\title{
Design of Steerable Wavelets to Detect Multifold Junctions
}

\author{
Zsuzsanna Püspöki, Student Member, IEEE, Virginie Uhlmann, Student Member, IEEE, \\ Cédric Vonesch, Member, IEEE, and Michael Unser, Fellow, IEEE
}

\begin{abstract}
We propose a framework for the detection of junctions in images. Although the detection of edges and key points is a well examined and described area, the multiscale detection of junction centers, especially for odd orders, poses a challenge in pattern analysis. The goal of this paper is to build optimal junction detectors based on 2D steerable wavelets that are polar-separable in the Fourier domain. The approaches we develop are general and can be used for the detection of arbitrary symmetric and asymmetric junctions. The backbone of our construction is a multiscale pyramid with a radial wavelet function where the directional components are represented by circular harmonics and encoded in a shaping matrix. We are able to detect $M$-fold junctions in different scales and orientations. We provide experimental results on both simulated and real data to demonstrate the effectiveness of the algorithm.
\end{abstract}

Index Terms-Symmetries, steerability, wavelet transform, junction detection.

\section{INTRODUCTION}

$\mathbf{L}$ OCAL junctions are common in nature. For instance, hexagonal patterns that correspond to three-fold vertices appear in structures such as the endothelial cells of the cornea, the organization of embryonic stem cells, and the cross section of honeycombs.

The key elements of those images are $M$-fold junctionsincluding the case $M=2$, which we identify as ridges. They support essential image-processing tasks such as cell segmentation, counting of cells, and image statistics. As an example, the accurate detection of cell structures (like tight junctions) that exhibit polygonal shapes is fundamental in stem cell research [2]. The application area of detecting junctions is continuously growing [3]-[7] as image analysis is becoming more relevant in data processing, but the difficulty in the detection of junctions is twofold. First, patterns in natural images are affected by unknown geometric transformations

Manuscript received March 1, 2015; revised October 14, 2015; accepted December 9, 2015. Date of publication December 11, 2015; date of current version December 28, 2015. This work was supported in part by the European Research Council under Grant ERC-2010-AdG 267439-FUN-SP, in part by the Center for Biomedical Imaging of the Geneva-Lausanne Universities, École polytechnique fédérale de Lausanne, Lausanne, Switzerland, and in part by the Hasler Foundation. The associate editor coordinating the review of this manuscript and approving it for publication was Prof. Béatrice Pesquet-Popescu.

The authors are with the Biomedical Imaging Group, École polytechnique fédérale de Lausanne, Lausanne CH-1015, Switzerland (e-mail: zsuzsanna. puspoki@epfl.ch; virginie.uhlmann@epfl.ch; $\quad$ cedric.vonesch@epfl.ch; michael.unser@epfl.ch).

Some preliminary results of this work appreared in [1]

Color versions of one or more of the figures in this paper are available online at http://ieeexplore.ieee.org.

Digital Object Identifier 10.1109/TIP.2015.2507981 such as (possibly local) rotation, translation, and scaling. Second, biological micrographs are frequently suffering from various types of distortion such as local variations in intensity or measurement noise.

Within the framework of 2D steerable wavelet frames, we propose a method to overcome these difficulties and to detect the location and orientation of junctions and local symmetries in an efficient way. We provide a multiscale detection scheme by the wavelet-type representation. We achieve angular selectivity by complementing the multiscale scheme with spatial rotation (steerability). Our methodology introduces a novel wavelet-design scheme along with an innovative detection algorithm based on analytical optimization. Our 2D steerable wavelets are polar-separable in the Fourier domain. The decomposition involves a pyramid characterized by a radial wavelet function and a set of directional components encoded using circular harmonics. The tightness of the generated frame ensures the perfect-reconstruction property.

\section{A. State of the Art}

In this work, we consider junctions and symmetry centers in a local sense, in opposition to global approaches, where the detection of the symmetry axis of different objects is targeted [8].

Existing solutions to the detection of junctions mostly fall into one of three categories: methods based on detection, grouping, and analysis of edges and gradients [9]; methods relying on feature analysis with structure tensors and their derivatives [10], [11]; and approaches based on template matching [12], [13], including some that use steerable filters [14]-[17].

Methods of the first two categories are often used to identify points of interest in computer-vision applications. They are typically adapted to differentiate between junctions, edges, or other varieties of key points. Several approaches rely on discrete, pixel-based models, such as mathematical morphology, or multiple directional non-maximum suppression [18]. The earliest and simplest techniques rely on gradient information. The definition of the gradient can be discretized and implemented easily for images, hence its popularity (e.g. Canny edge detector [19]). However, the orientation estimation is very sensitive to noise.

To capture higher-order directional structures, the gradient information is replaced by higher-order derivatives. For instance, Xia et al. [4] are using a template-free method for 
the detection and grouping of junctions based on the strength of directional derivatives.

Since the pioneering work of Förstner [20] and Harris and Stephens [21], the structure tensor, which can be interpreted as a localised covariance matrix of the gradient, has become a popular tool for low-level feature analysis, and in particular for corner and ridge detection. Further improvements of this method can be found in [10] and [11].

For junction and line detection, it is often desirable to design linear filters that respond exclusively to structures within a narrow angular range. In order to construct such filters, Fourier-domain designs are very powerful. Typically, one constructs filters that are supported on wedges in the Fourier domain.

Directional selectivity can be augmented by multiresolution methods, typically using wavelets. This makes it possible to process oriented features independently at different scales, e.g., only for coarse or fine structures. Classical examples of this type are Gabor wavelets [22], which cover the frequency plane using Gaussian windows to approximate a (rescaled) partition of unity [23]. They can serve as a model for the impulse response of the filters in the mammalian visual system [24]. Cauchy wavelets have also been used for symmetry detection [25].

Kovesi accounts for detecting symmetries and anti-symmetries based on local phase information provided by wavelets constructed with complex valued Log Gabor functions [26]. However, his work is mostly focusing on bilateral symmetries and ignores rotational ones.

Over the last decade, curvelets [27], shearlets [28], [29] and contourlets [30] have attracted a lot of interest. Their key property and main difference with respect to directional wavelets is that they increase their directionality as the scale gets finer. Curvelets approximate rotation and dilation invariance by using a set of basis functions from a series of rotated and dilated versions of an anisotropic mother wavelet. Contourlets reproduce the same frequency partitioning as curvelets, based on a tree-structured filterbank. Shearlets are designed in the discrete Fourier domain to achieve exact rotation invariance. They are well suited for the analysis and synthesis of images with highly directional features. Examples of particular applications can be found in [31] and [32]. A thorough description of wavelets, contourlets, shearlets and other related transforms can be found in [33]. The importance of these tools in image analysis and a comparison between each of them is discussed in [34].

Typically, methods for junction detection involves detectors with parametric templates corresponding to specific types of junctions. With the exception of steerable filters/wavelets, these approaches generally involve discretizing rotation angles, which entails a tradeoff between angular precision and computational cost of detection.

Müehlich et al. [17] proposed a combined method for the detection and classification of polar separable patterns (including junctions). The detection phase relies on a classical structure tensor scheme, complemented by further operations to truncate the list of candidate points. The classification step is dedicated to the determination of the precise angular orientation of the "branches". For such purpose, multisteerable filters are designed and adjusted to the patterns of interest.

All existing approaches, including the ones using steerable filters, are focused on detection, most often of one specific pattern. Unlike the wavelet frames proposed in the present work, earlier methods fail to provide a framework for the multiscale decomposition and analysis/synthesis of images based on junctions and centers of local symmetry. In comparison, the $M$-fold tight wavelet frames we propose facilitate the detection and analysis of features at different scales, can deal with arbitrary (continuous) rotations in an efficient and systematic manner, and enable an energy-based analysis and image reconstruction thanks to the tight-frame property.

\section{B. Roadmap}

The paper is organized as follows: In Section 2, we recall the principle of steerability and the construction of steerable wavelet frames, which provide the analytical framework used in this work. Then, in Section 3, we examine the design of $M$-fold symmetric steerable wavelet frames both in the space and the Fourier domains. We propose in Section 4 a general framework to design $M$-fold detectors relying on a unimodal detector. In Section 5, we show that our symmetric steerable wavelet design can be generalized to other classes of local structures and junctions. In Section 6, we evaluate the different design methods based on the ease of generation of symmetric patterns. We then present in Section 7 an algorithm to detect centers of symmetry. We finally apply our method to synthetic and real biological micrographs in Section 8.

\section{Steerability and Steerable WaVelet Frames}

\section{A. Notation}

The Fourier transform of a function $f \in L_{1}\left(\mathbb{R}^{2}\right)$, is denoted by $\mathscr{F}\{f\}=\widehat{f}$ and computed as

$$
\widehat{f}(\boldsymbol{\omega})=\int_{\mathbb{R}^{2}} f(\boldsymbol{x}) \mathrm{e}^{-\mathrm{j}\langle\boldsymbol{x}, \boldsymbol{\omega}\rangle} \mathrm{d} \boldsymbol{x} .
$$

The Cartesian and polar representations of the 2D function $f$ are denoted by $f(\boldsymbol{x})$ with $\boldsymbol{x} \in \mathbb{R}^{2}$ and $f_{\text {pol }}(r, \theta)$ with $r \in \mathbb{R}^{+}$, $\theta \in[0,2 \pi)$. We may drop the 'pol' subscript where it can be understood from the context. Similarly, in the Fourier domain, we write $\hat{f}(\omega)$ and $\hat{f}_{\text {pol }}(\rho, \varphi)$ with $\omega \in \mathbb{R}^{2}$ and $\rho \in \mathbb{R}^{+}$, $\varphi \in[0,2 \pi)$.

\section{B. Steerable Functions}

The basics of steerability were formulated by Freeman and Adelson [35] in the early nineties [35], and developed further by Perona [36], Simoncelli and Farid [15], Unser and Chenouard [37], and Ward and Unser [38]. We define steerable wavelet frames according to [14] and present the parametric framework for 2D steerable wavelet transforms along the lines of [37].

Definition 1: A function $f$ on the plane is steerable in the finite basis $\left\{f^{(1)}, \ldots, f^{(N)}\right\}$ if, for any rotation matrix $\mathbf{R}_{\theta}$, we can find coefficients $c_{1}(\theta), \ldots, c_{N}(\theta)$ such that

$$
f\left(\mathbf{R}_{\theta} \boldsymbol{x}\right)=\sum_{n=1}^{N} c_{n}(\theta) f_{n}(\boldsymbol{x})
$$


Definition 2: A family of functions $\left\{f^{(1)}, \ldots, f^{(N)}\right\}$ is steerable if its linear span is invariant under arbitrary rotations.

It means that any rotation of $f$ can be expanded in the same finite basis, up to a systematic adaptation of the coefficients. The simplest nontrivial example is the function with polar representation $f_{\mathrm{pol}}(r, \theta)=\cos (\theta)$, where the rotation of $f_{\text {pol }}(r, \theta)$ by $\theta_{0}$ can be written as $\cos \left(\theta+\theta_{0}\right)=$ $\cos \left(\theta_{0}\right) \cos (\theta)-\sin \left(\theta_{0}\right) \sin (\theta)$, which is a weighted sum of $f^{(1)}(\theta)=\cos (\theta)$ and $f^{(2)}(\theta)=\sin (\theta)$. Furthermore, the polar-separable function $f_{\mathrm{pol}}(r, \theta)=\eta(r) \mathrm{e}^{\mathrm{j} \theta}$, where the angular part is a pure complex exponential, is also steerable since $f_{\text {pol }}\left(r, \theta+\theta_{0}\right)=\mathrm{e}^{\mathrm{j} \theta_{0}} f_{\mathrm{pol}}(r, \theta)$. Consequently, if

$$
f_{\mathrm{pol}}(r, \theta)=\sum_{n \in S} u_{n} f_{\mathrm{pol}}^{(n)}(r, \theta)
$$

for some finite set $S \subset \mathbb{Z}$, where $f_{\mathrm{pol}}^{(n)}(r, \theta)$ is polarseparable as

$$
f_{\text {pol }}^{(n)}(r, \theta)=\eta_{n}(r) \mathrm{e}^{\mathrm{j} n \theta},
$$

then

$$
f_{\text {pol }}\left(r, \theta+\theta_{0}\right)=\sum_{n \in S} \mathrm{e}^{\mathrm{j} n \theta_{0}} u_{n} f_{\text {pol }}^{(n)}(r, \theta) .
$$

This means that any function $f$ of the above form is steerable, and the steered coefficients for $\theta_{0}$ are obtained from the original ones by multiplication with $\mathrm{e}^{\mathrm{j} n \theta_{0}}$. This fact has an important role in the design of steerable wavelet frames.

\section{Circular Harmonic Wavelet Frames}

To capture the local orientation of features in an image within a multiresolution hierarchy, we combine the concept of steerability with the concept of a tight wavelet frame. To that end, we construct a wavelet frame consisting of shifted and scaled versions of steerable functions. Proposition 1 states sufficient conditions for such a wavelet system.

Proposition 1 (cf. [37, Proposition 4.1.]): Let $h:[0, \infty) \rightarrow$ $\mathbb{R}$ be a smooth function satisfying

1) $\hat{h}(\rho)=0$ for $|\rho|>\pi \quad$ (bandlimited)

2) $\sum_{s \in \mathbb{Z}}\left|\hat{h}\left(2^{s} \rho\right)\right|^{2}=1$

3) $\left.\frac{\mathrm{d}^{n} \hat{h}}{\mathrm{~d} \rho^{n}}\right|_{\rho=0}=0$ for $n=0, \ldots, N$ (vanishing moments).

For $1 \leq p \leq \infty$, the mother wavelet $\phi$ with Fourier transform

$$
\widehat{\phi}(\omega)=\hat{h}\left(\|\omega\|_{\ell_{p}}\right)
$$

generates a normalized tight wavelet frame of $L_{2}\left(\mathbb{R}^{d}\right)$ whose basis functions

$$
\phi_{s, \boldsymbol{k}}(\boldsymbol{x})=\phi\left(\boldsymbol{x}-2^{s} \boldsymbol{k}\right)
$$

have vanishing moments up to order $N$. In particular, any $f \in L_{2}\left(\mathbb{R}^{d}\right)$ can be represented as

$$
f=\sum_{s \in \mathbb{Z}} \sum_{\boldsymbol{k} \in \mathbb{Z}^{d}}\left\langle f, \phi_{s, \boldsymbol{k}}\right\rangle \phi_{s, \boldsymbol{k}} .
$$

There are several primal profiles satisfying Proposition 1 . In this work we use Simoncelli's isotropic wavelet [39] since it is inspired from biological vision and has several wellestablished applications in image analysis. It is defined by its radial frequency profile

$$
\hat{h}(\|\omega\|)= \begin{cases}\cos \left(\frac{\pi}{2} \log _{2}\left(\frac{2\|\omega\|}{\pi}\right)\right), & \frac{\pi}{4}<\|\omega\| \leq \pi \\ 0, & \text { otherwise. }\end{cases}
$$

We start our design from a bandlimited isotropic mother wavelet $h$ over $\mathbb{R}^{2}$ that is such that its shifts and dilations form a wavelet frame. The isotropic wavelet at scale $s \in \mathbb{Z}$ and location (grid point) $\boldsymbol{x}_{\boldsymbol{k}}=2^{s} \boldsymbol{k}, \boldsymbol{k} \in \mathbb{Z}^{2}$ has the form

$$
h_{s, k}(\boldsymbol{x})=h_{s}\left(\boldsymbol{x}-\boldsymbol{x}_{\boldsymbol{k}}\right)=\frac{1}{2^{s}} h\left(\frac{\boldsymbol{x}-\boldsymbol{x}_{k}}{2^{s}}\right)=\frac{1}{2^{s}} h\left(\frac{\boldsymbol{x}}{2^{s}}-\boldsymbol{k}\right),
$$

or, in the Fourier domain,

$$
\left.h_{s} \widehat{\left(\cdot-x_{k}\right.}\right)(\omega)=2^{s} \hat{h}\left(2^{s} \omega\right) \mathrm{e}^{-\mathrm{j}\left\langle\boldsymbol{x}_{k}, \boldsymbol{\omega}\right\rangle},
$$

and, in polar coordinates,

$$
\hat{h}_{s, \boldsymbol{k}}(\rho, \varphi)=2^{s} \hat{h}\left(2^{s} \rho\right) \mathrm{e}^{-\mathrm{j} \rho_{\boldsymbol{k}} \rho \cos \left(\varphi-\varphi_{\boldsymbol{k}}\right)},
$$

with $\boldsymbol{x}_{\boldsymbol{k}}=\rho_{\boldsymbol{k}} \mathrm{e}^{\mathrm{j} \varphi_{k}}$ in polar form.

To utilize the idea presented in Section II.B., we use exponentials to define the $N$-channel tight frame of wavelets $\left\{\xi_{s, \boldsymbol{k}}^{(n)}\right\}=\left\{\mathscr{F}^{-1}\left\{\hat{\xi}_{s, \boldsymbol{k}}^{(n)}\right\}\right\}$ by

$$
\begin{aligned}
\hat{\xi}_{s, \boldsymbol{k}}^{(n)}(\rho, \varphi) & =\mathrm{e}^{\mathrm{j} n \varphi} \hat{h}_{s, \boldsymbol{k}}(\rho, \varphi) \\
& =2^{s} \hat{h}\left(2^{s} \rho\right) \mathrm{e}^{\mathrm{j} n \varphi-\mathrm{j} \rho_{\boldsymbol{k}} \rho \cos \left(\varphi-\varphi_{k}\right)},
\end{aligned}
$$

where the values of $n$ are distinct and taken from a predefined set $S=\left\{n_{1}, \ldots, n_{N}\right\}$ called the set of harmonics. The steerable property of the wavelet is satisfied, since they take the form of (4) (see Proposition 2 below). For a proof of the fact that this transformation maintains the wavelet frame equality of the original wavelet frame, we refer to [37].

We note that, in the Fourier domain, $\hat{\xi}_{s, \boldsymbol{k}}^{(n)}(\rho, \varphi)$ can be generated as the $n$ th-order complex Riesz transform of $\hat{h}_{s, \boldsymbol{k}}(\rho, \varphi)$. The $n$ th-order complex Riesz transform $\mathscr{R}^{n}$ is defined in the Fourier domain as

$$
\mathscr{F}\left\{\mathscr{R}^{n}\{f\}\right\}(\rho \cos \varphi, \rho \sin \varphi)=\mathrm{e}^{\mathrm{j} n \varphi} \hat{f}_{\mathrm{pol}}(\rho, \varphi),
$$

and corresponds to the $n$-fold iterate of the complex Riesz transform $\mathscr{R}$. The Riesz transform is scale- and shift-invariant,

$$
\begin{aligned}
\forall x_{0} \in \mathbb{R}^{d}, \quad \mathscr{R}\left\{f\left(\cdot-x_{0}\right)\right\}(x) & =\mathscr{R} f\left(x-x_{0}\right) \\
\forall a \in \mathbb{R}^{+} \backslash\{0\}, \quad \mathscr{R}\left\{f\left(\frac{\dot{a}}{a}\right)\right\}(\boldsymbol{x}) & =\mathscr{R} f\left(\frac{x}{a}\right),
\end{aligned}
$$

and provides a unitary mapping from $L_{2}\left(\mathbb{R}^{2}\right)$ to $L_{2}\left(\mathbb{R}^{2}\right)$. The Riesz transform commutes with spatial rotations, in the sense that its impulse response is steerable,

$$
\mathscr{R}\{\delta\}\left(\boldsymbol{R}_{\theta_{0}} \boldsymbol{x}\right)=\mathrm{e}^{-\mathrm{j} \theta_{0}} \mathscr{R}\{\delta\}(\boldsymbol{x}),
$$

where $\boldsymbol{R}_{\theta_{0}}=\left[\begin{array}{cc}\cos \theta_{0} & -\sin \theta_{0} \\ \sin \theta_{0} & \cos \theta_{0}\end{array}\right]$ is the matrix that implements a $2 \mathrm{D}$ spatial rotation by the angle $\theta_{0}$. These properties are preserved through iteration, thus they are valid for the $n$ th-order complex Riesz transform as well. 
Due to the correspondence between rotations in space and Fourier domain, the wavelets centered around $\boldsymbol{x}_{\boldsymbol{k}}=\mathbf{0}$ can be steered to an angle $\varphi_{0}$ by multiplication with $\mathrm{e}^{\mathrm{j} n \varphi_{0}}$. We note that an $n$ th-order harmonic wavelet has a rotational symmetry of order $n$ around its center, corresponding to the $n$ th-order rotational symmetry of $\mathrm{e}^{\mathrm{j} n \varphi}$. The tight frame of steerable wavelets defined by the functions $\xi_{s, \boldsymbol{k}}^{(n)}$ is referred to as circular harmonic wavelets [40].

The properties of steerable filters using low-order harmonics are analyzed in [41]. A general design of steerable filters for feature detection was presented in [42]. The relation between the Riesz transform and steerable filters is presented in [43]. The connection between wavelet steerability and the highorder Riesz transform is discussed in [44]. An application of steerable Riesz wavelets for texture learning was presented in [45]. Spherical harmonics, which are the 3D counterparts of circular harmonics, have also been used to represent and detect features and shapes in 3D [46], [47]. There, the authors are looking for symmetric structures; however, they apply it to an entire 3D shape, not locally. Another related publication is [48] where the authors rely on the Riesz transform to analyze predefined junction points in images.

\section{Other Representations of Steerable Wavelets}

We take advantage of the circular harmonic wavelets defined in Section II. C. to design wavelets and construct new steerable representations that detect features of interest at scale $s$ and location $\boldsymbol{x}_{\boldsymbol{k}}$. The new steerable frame functions are obtained by a mapping with the orthogonal shaping matrix $\mathbf{U}$ that is such that

$$
\left[\begin{array}{c}
\psi_{s, \boldsymbol{k}}^{\left(n_{1}\right)} \\
\vdots \\
\psi_{s, \boldsymbol{k}}^{\left(n_{N}\right)}
\end{array}\right]=\mathbf{U}\left[\begin{array}{c}
\xi_{s, \boldsymbol{k}}^{\left(n_{1}\right)} \\
\vdots \\
\xi_{s, \boldsymbol{k}}^{\left(n_{N}\right)}
\end{array}\right] .
$$

The new wavelets span the same space as the wavelet frame $\left\{\xi_{s, k}^{(n)}\right\}$. The shaping matrix $\mathbf{U}$ endows the wavelet functions $\psi_{s, \boldsymbol{k}}^{(n)}$ with a desired angular profile.

There are several ways to design the shaping matrix. We propose to define an angular energy concentration like $\sum_{n} u_{n} \xi^{(n)}$ (corresponding to a single row of $\mathbf{U}$, thus to a single wavelet) and to optimize it with respect to the angular weights $u_{n}$ that control the concentration of the energy along the desired pattern, with $\boldsymbol{u}=\left(u_{1}, u_{2}, \ldots, u_{N}\right)$ and $\boldsymbol{u}^{\mathrm{H}} \boldsymbol{u}=1$. This formulation is reminiscent of what appears in the works of Slepian [49]. Its solution is found similarly through an eigen decomposition.

We can formulate this energy optimization either in the spatial or in the Fourier domain. As we shall see shortly, both designs are appropriate to generate symmetric junction templates; however, each one has its own advantages and disadvantages. We examine and discuss both cases in Sections III.A and III.B, respectively.

\section{Design OF $M$-Fold-S YMmetric WaVElets}

\section{A. Steerable Wavelet Design in the Space Domain}

To design a wavelet that responds to $M$-fold symmetries, we propose to impose the same $M$-fold-symmetric pattern on $\psi$. We achieve this by minimizing a well chosen energy functional. As an illustration, in the case of a unimodal detector, we are looking for the angular profile that is most concentrated around the angle 0 . This means that we are targeting a minimum-variance solution, which results in an energy that concentrates around a chosen axis.

The spatial quadratic energy term we propose to minimize has the form

$$
E\{\psi\}=\frac{1}{2 \pi} \int_{0}^{\infty} \int_{-\pi}^{\pi}|\psi(r, \theta)|^{2} w(\theta) \mathrm{d} \theta r \mathrm{~d} r,
$$

where $w$ is an $M$-fold-symmetric nonnegative weighting function with $M$ equidistant minima on the unit circle that favors $M$-fold-symmetric solutions. Minimizing $E$ will thus force the solution $\psi$ to be localized symmetrically near the $M$-fold minima. Once the mother wavelet $\psi$ is found, its translates and dilates will naturally share the optimal angular profile around their center.

By expanding $\psi$ as $\sum_{n} u_{n} \xi^{(n)}$ and imposing a unit norm on $\boldsymbol{u}$, this formalism boils down to a quadratic optimization problem with quadratic constraints that can be solved through an eigen decomposition. During the minimization process, the radial part of the wavelet function results in Hankel-like integrals. We take advantage of Proposition 2 to determine the quadratic optimization problem.

Proposition 2: If the Fourier transform $\hat{\xi}^{(n)}(\rho, \varphi)$ of a wavelet function is polar-separable in the Fourier domain and its angular part is a pure exponential term $\left(\hat{\xi}^{(n)}(\rho, \varphi)=\hat{h}(\rho) \mathrm{e}^{\mathrm{j} n \varphi}\right)$, then its inverse Fourier transform $\mathscr{F}^{-1}\left\{\hat{\xi}^{(n)}(\rho, \varphi)\right\}$ is polar-separable in the space domain and takes the form

$$
\xi^{(n)}(r, \theta)=\eta_{n}(r) \mathrm{e}^{\mathrm{j} n \theta}
$$

where

$$
\eta_{n}(r)=\frac{\mathrm{j}^{n}}{2 \pi} \int_{0}^{\infty} \rho \hat{h}(\rho) \mathbf{J}_{n}(r \rho) \mathrm{d} \rho,
$$

with $\mathbf{J}_{n}$ denoting the nth Bessel function of the first kind.

(The proof of Proposition 2 is given in Appendix A.) By replacing $\sum_{n \in S} u_{n} \xi^{(n)}(r, \theta)=\sum_{n \in S} u_{n} \eta_{n}(r) \mathrm{e}^{\mathrm{j} n \theta}$ for $\psi(r, \theta)$ in the energy functional, we arrive at the form

$$
E\{\psi\}=\boldsymbol{u}^{\mathrm{H}} \mathbf{W} \boldsymbol{u},
$$

where the entries of $\mathbf{W}$ are given by

$$
\mathbf{W}_{n, n^{\prime}}=\frac{1}{2 \pi} \int_{-\pi}^{\pi} \mathrm{e}^{\mathrm{j}\left(n-n^{\prime}\right) \theta} w(\theta) \mathrm{d} \theta \int_{0}^{\infty} \eta_{n}(r) \overline{\eta_{n^{\prime}}(r)} r \mathrm{~d} r .
$$

The radial and angular factors are computed separately. The angular factor

$$
W\left(n-n^{\prime}\right)=\frac{1}{2 \pi} \int_{-\pi}^{\pi} \mathrm{e}^{\mathrm{j}\left(n-n^{\prime}\right) \theta} w(\theta) \mathrm{d} \theta
$$

is the $\left(n-n^{\prime}\right)$ th Fourier coefficient of $w(\theta)$, while the radial part

$$
\begin{aligned}
H_{\mathrm{sp} .}\left(n, n^{\prime}\right)= & \int_{0}^{\infty} \eta_{n}(r) \overline{\eta_{n^{\prime}}(r)} r \mathrm{~d} r \\
= & \int_{0}^{\infty}\left(\int_{0}^{\infty} \rho \hat{h}(\rho) \mathrm{J}_{n}(r \rho) \mathrm{d} \rho\right) \\
& \times\left(\int_{0}^{\infty} \rho \overline{\hat{h}(\rho)} \mathrm{J}_{n^{\prime}}(r \rho) \mathrm{d} \rho\right) r \mathrm{~d} r
\end{aligned}
$$



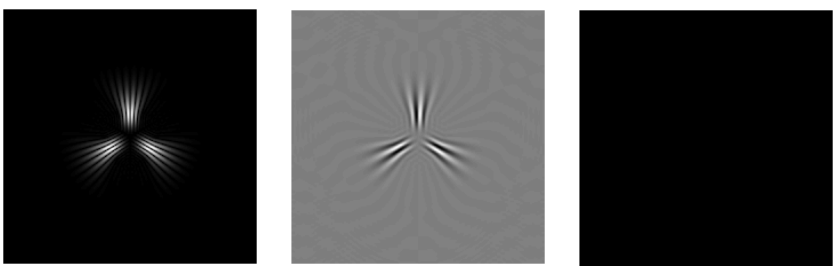

Fig. 1. Three-fold-symmetric wavelet designed by the energy functional in the space domain and visualized in the space domain. From left to right: magnitude, real, and imaginary parts, respectively. Harmonics: $S=\{3 n: n=-8, \ldots, 8\}$.
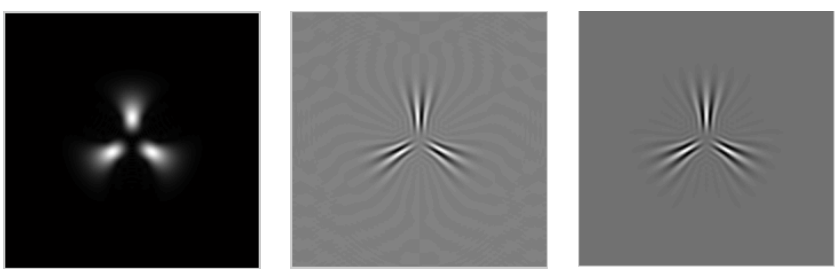

Fig. 2. Three-fold-symmetric complex wavelet designed by the energy functional in the space domain and visualized in the space domain. From left to right: magnitude, real, and imaginary parts, respectively. Harmonics: $S=\{3 n: n=0, \ldots, 9\}$.

is a multiplicative term that corresponds to the effect of the radial part. Finally, the entries of $\mathbf{W}$ are computed as

$$
\mathbf{W}_{n, n^{\prime}}=H_{\text {sp. }}\left(n, n^{\prime}\right) W\left(n-n^{\prime}\right) .
$$

It turns out that $\mathbf{W}$ is a Hermitian-symmetric matrix with entries indexed by $n, n^{\prime} \in S$.

With the previous derivation, we end up with a quadratic optimization problem (minimization of $E=\boldsymbol{u}^{\mathrm{H}} \mathbf{W} \boldsymbol{u}$ ) with quadratic constraints $\left(\boldsymbol{u}^{\mathrm{H}} \boldsymbol{u}=1\right)$, which is a standard eigenvalue problem. Minimizing the energy functional is equivalent to finding the eigenvector that corresponds to the smallest eigenvalue of $\mathbf{W}$. We note that the matrix $\mathbf{W}$ is positive-semi-definite since it is derived from a nonnegative weight function, which itself defines a nonnegative energy (Bochner's theorem).

A particular example of a three-fold-symmetric wavelet obtained by the proposed method can be seen in Figure 1. The weight function $w$ is $\frac{2 \pi}{3}$-periodic, with $w(\theta)=\theta^{2}$ for $|\varphi| \leq \frac{\pi}{3}$ to achieve maximal energy concentration about the minima in the sense of variance. An example of a complex threefold-symmetric wavelet obtained by the proposed method is presented in Figure 2.

\section{B. Design of Steerable Wavelets in the Fourier Domain}

Since rotations and symmetries in the space domain carry over to the frequency domain, we can formulate our minimization problem in either one. The advantage of the frequency-domain formulation is to avoid the computation of Hankel-like integrals. This happens because, in the Fourier domain, the radial part of the wavelet has no effect on the optimization.

The Fourier-domain energy functional $E$ is defined as

$$
E\{\hat{\psi}\}=\frac{1}{2 \pi} \int_{0}^{\infty} \int_{-\pi}^{+\pi}\|\hat{\psi}(\rho, \varphi)\|^{2} w(\varphi) \mathrm{d} \varphi \rho \mathrm{d} \rho,
$$

where $w \geq 0$ is an $M$-fold-symmetric weighting function with $M$ equidistant minima on the unit circle. As stated in
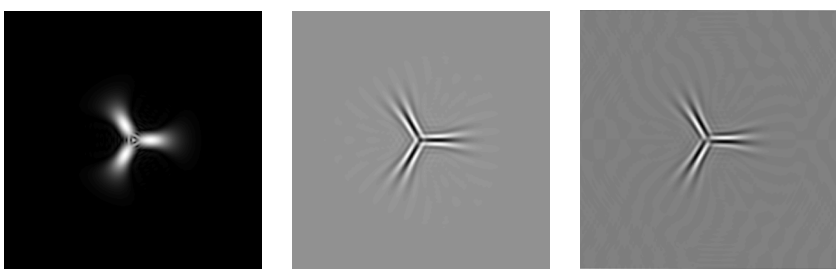

Fig. 3. Three-fold-symmetric wavelet designed by the energy functional in the Fourier domain and visualized in the space domain. From left to right: magnitude, real, and imaginary parts, respectively. Harmonics: $S=\{3 n: n=0, \ldots, 9\}$.

the Section III.A, minimizing $E$ will force the solution $\hat{\psi}$ to be localized symmetrically near the $M$-fold minima.

The polar factorization of $\hat{\psi}$ can be written as

$$
\hat{\psi}(\rho, \varphi)=\sum_{n \in S} u_{n} \hat{\xi}^{(n)}(\rho, \varphi)=\hat{h}(\rho) \sum_{n \in S} u_{n} \mathrm{e}^{\mathrm{j} n \varphi} .
$$

The energy functional (27) therefore factorizes as

$$
E\{\hat{\psi}\}=\int_{0}^{\infty}|\hat{h}(\rho)|^{2} \rho \mathrm{d} \rho \cdot \frac{1}{2 \pi} \int_{-\pi}^{\pi}\left|\sum_{n \in S} u_{n} \mathrm{e}^{\mathrm{j} n \varphi}\right|^{2} w(\varphi) \mathrm{d} \varphi .
$$

This means that the radial part of the wavelet function has no role in the optimization problem since it is factored out from the integral. As a result, we can optimize the angular profile of the wavelet by defining the Fourier-based energy for the angular factor alone as

$$
E_{\text {ang }}\{\hat{\psi}\}=\frac{1}{2 \pi} \int_{-\pi}^{\pi}\left|\sum_{n \in S} u_{n} \mathrm{e}^{\mathrm{j} n \varphi}\right|^{2} w(\varphi) \mathrm{d} \varphi .
$$

Consequently, formulating the energy term in the Fourier domain results in a substantial simplification of the problem. After replacing $\hat{\psi}(\varphi)$ by its expansion $\sum_{n \in S} u_{n} \mathrm{e}^{\mathrm{j} n \varphi}$, we have that

$$
\begin{aligned}
E_{\text {ang }}\{\hat{\psi}\} & =\sum_{n^{\prime} \in S} \sum_{n \in S} \overline{u_{n}^{\prime}} u_{n} \frac{1}{2 \pi} \int_{-\pi}^{+\pi} \mathrm{e}^{\mathrm{j}\left(n-n^{\prime}\right) \varphi} w(\varphi) \mathrm{d} \varphi \\
& =\sum_{n^{\prime} \in S} \sum_{n \in S} \overline{u_{n}^{\prime}} W\left(n-n^{\prime}\right) u_{n}=\boldsymbol{u}^{\mathrm{H}} \mathbf{W} \boldsymbol{u},
\end{aligned}
$$

where

$$
W\left(n-n^{\prime}\right)=\frac{1}{2 \pi} \int_{-\pi}^{+\pi} \mathrm{e}^{\mathrm{j}\left(n-n^{\prime}\right) \varphi} w(\varphi) \mathrm{d} \varphi
$$

is the $\left(n^{\prime}-n\right)$ th Fourier-series coefficient of $w$ (as in (24)) and $\mathbf{W}$ is the corresponding Hermitian-symmetric matrix with entries indexed by $n, n^{\prime} \in S$. Finally, and similarly to the space-domain design, we end up with an eigenvalue problem.

A particular example of a three-fold-symmetric wavelet obtained by the proposed method can be seen in Figure 3 . The weight function $w$ is $\frac{2 \pi}{M}$-periodic, with $w(\varphi)=\varphi^{2}$ for $|\varphi| \leq \frac{\pi}{M}$ to achieve maximal energy concentration about the minima in the sense of variance. Examples of higher-foldsymmetric wavelets can be seen in Figure 4.

For implementation purposes, the wavelets are projected on bandlimited basis functions that have a certain cutoff frequency. This suppresses all patterns above that frequency and explains why the center of our wavelets vanish. This effect can 

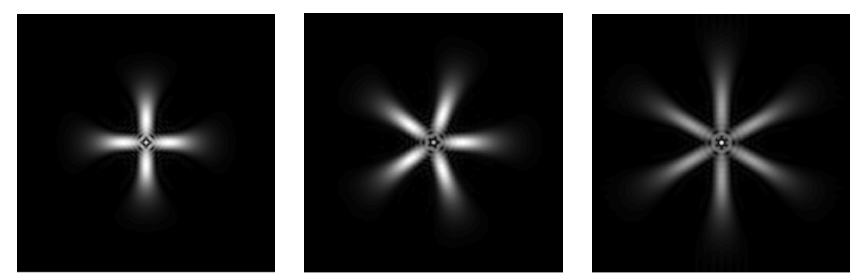

Fig. 4. Modulus of four-fold-, five-fold-, and six-fold-symmetric wavelets designed by the energy functional in the Fourier domain and visualized in the space domain. Harmonics: $S=\{M n: n=0, \ldots, 9\}$.

be seen in Figures 1 and 2. When the number of harmonics is high, we do not have enough bandwidth to represent the directional patterns within a disk of radius $R$. (Based on the Shannon-Nyquist sampling theorem, this occurs when $R<\frac{\sqrt{2}}{\pi N}$, where $N$ is the highest harmonic.) This phenomenon can be seen in Figure 3 at the center of the wavelet. However, we note that i) this phenomenon does not reduce the detection property of the wavelets; ii) one typically does not apply a large number of harmonics, also due to computational cost.

\section{Angular Weights And Asymmetric WaVelets}

Our framework is flexible and easy to generalize to many classes of local structures and junctions. Here, we take another look at the angular weighting functions and propose to design asymmetric wavelets, targeting familiar shapes like corners or T-junctions. The advantage, as before, is that we can "steer" these wavelets to any arbitrary angle by a systematic complex rescaling of the wavelet coefficients. The design of asymmetric wavelets is possible only in the space domain, since the rotational equivalence of the space and Fourier domains is now lost.

We begin our formulation with (26), by recalling the nature of the angular weighting function for the symmetric case. Then we adapt the angular factor $W$ and note that it corresponds to the (time-reversed) Fourier series of the angular weight function $w$. Thus, we achieve the desired shape by choosing $w$ such that its local extrema are placed at angles that correspond to the desired branches of the wavelet.

In the case of the symmetric three-fold junction of Section III.A and III.B, we had identified a profile that, after proper steering, was simultaneously concentrated around zero, $\frac{2 \pi}{3}$, and $\frac{-2 \pi}{3}$. By the energy-minimization paradigm, we now choose $w$ such that it also has three identical minima at 0 , $\frac{2 \pi}{3}$, and $\frac{-2 \pi}{3}$. This function is given by the parabola $\theta^{2}$ over the interval $[-\pi / 3, \pi / 3]$, and its translates to the intervals $[\pi / 3, \pi]$ and $[-\pi / 3,-\pi]$. The function $w_{S 3}$ on the interval $[-\pi, \pi]$ is illustrated in Figure 5. More precisely,

$$
\begin{gathered}
w_{S 3}(\theta)=C_{S 3}\left(\theta^{2} \mathbf{1}_{[-\pi / 3, \pi / 3]}(\theta)+(\theta-2 \pi / 3)^{2} \mathbf{1}_{[\pi / 3, \pi]}(\theta)\right. \\
\left.+(\theta+2 \pi / 3)^{2} \mathbf{1}_{[-\pi,-\pi / 3]}(\theta)\right)
\end{gathered}
$$

Here, $C_{S 3}$ is the normalization constant $(3 / \pi)^{2}$. The corresponding kernel is

$$
\begin{aligned}
& W_{S 3}(q)=C_{S 3} \\
& \times \frac{\left(1+2 \cos \left(\frac{2 \pi}{3} q\right)\right)\left(\left(\pi^{2} q^{2}-18\right) \sin \left(\frac{\pi}{3} q\right)+6 \pi q \cos \left(\frac{\pi}{3} q\right)\right)}{9 q^{3} \pi} .
\end{aligned}
$$

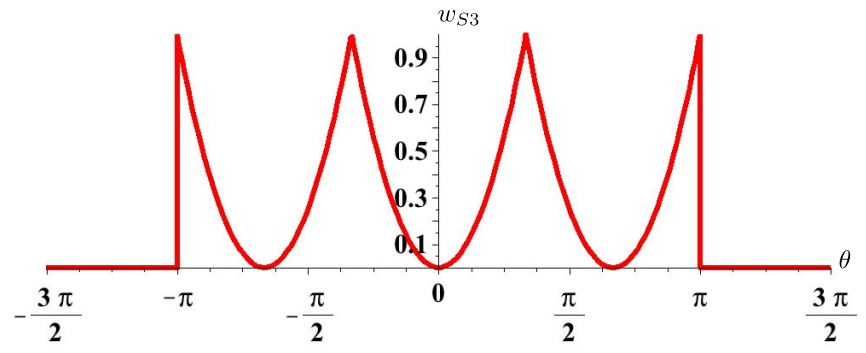

Fig. 5. Angular weighting function for the design of symmetric wavelets (three-fold-symmetric shape).

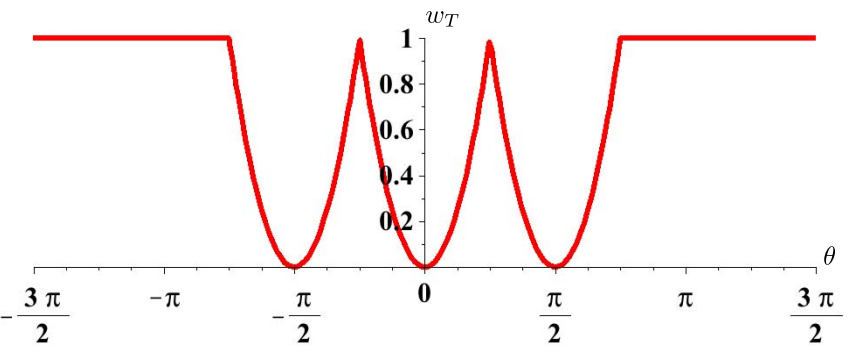

Fig. 6. Angular weighting function for the design of asymmetric wavelets ( $\mathrm{T}$ shape).

In the case of a $\mathrm{T}$ junction, we intend to identify profiles that, after proper steering, are simultaneously concentrated around zero, $\pi / 2$, and $-\pi / 2$. Similarly to the symmetric case, using the energy minimization paradigm, we take $w$ to have three identical minima at $0, \pi / 2$, and $-\pi / 2$, given by the parabola $\theta^{2}$ over the interval $[-\pi / 4, \pi / 4]$ and its translates to the intervals $[\pi / 4,3 \pi / 4]$ and $[-3 \pi / 4,-\pi / 4]$. We keep $w_{T}$ constant over the intervals $[3 \pi / 4, \pi]$ and $[-\pi,-3 \pi / 4]$. The function $w_{T}$ on the interval $[-\pi, \pi]$ is illustrated in Figure 6. Specifically,

$$
\begin{aligned}
w_{T}(\theta)= & C_{T}\left(\theta^{2} \mathbf{1}_{[-\pi / 4, \pi / 4]}(\theta)+(\theta-\pi / 2)^{2} \mathbf{1}_{[\pi / 4,3 \pi / 4]}(\theta)\right. \\
& \left.+(\theta+\pi / 2)^{2} \mathbf{1}_{[-3 \pi / 4,-\pi / 4]}(\theta)\right) \\
& +\mathbf{1}_{[-\pi,-3 \pi / 4]}(\theta)+\mathbf{1}_{[3 \pi / 4, \pi]}(\theta),
\end{aligned}
$$

where $C_{T}$ is the normalization constant $(4 / \pi)^{2}$. The corresponding kernel is

$$
\begin{aligned}
& W_{T}(q) \\
& =\frac{\pi^{2} q^{2} \sin (\pi q)+8 \pi q\left(\cos \left(\frac{3 \pi}{4} q\right)+2 \cos \left(\frac{\pi}{4} q\right)\right)-32 \sin \left(\frac{3 \pi}{4} q\right)}{\pi^{3} q^{3}} .
\end{aligned}
$$

The wavelet obtained by the proposed method and its weight function can be seen in Figures 6 and 8 .

Similarly, for the perpendicular corner, the weight function consists of two identical parabolas placed at $-\pi / 4$ and $\pi / 4$. We keep $w_{C}$ constant over the intervals $[\pi / 2, \pi]$ and $[-\pi,-\pi / 2]$. This leads to the kernel illustrated in Figure 7 with

$$
\begin{aligned}
w_{C}(\theta)= & C_{C}\left((\theta-\pi / 4)^{2} \mathbf{1}_{[0, \pi / 2]}(\theta)+(\theta+\pi / 4)^{2} \mathbf{1}_{[-\pi / 2,0]}(\theta)\right) \\
& +\mathbf{1}_{[-\pi,-\pi / 2]}(\theta)+\mathbf{1}_{[\pi / 2, \pi]}(\theta),
\end{aligned}
$$


TABLE I

COMPARISON OF DESIGNS

\begin{tabular}{l|l|l}
\hline \hline Property & Fourier-domain design & Space-domain design \\
\hline Real odd symmetric wavelets & Not possible & Possible \\
Design of asymmetric wavelets & Not possible & Possible \\
Computational complexity & Analytical results & Numerical integration needed \\
Ease of design & Fast & Slower \\
Design of arbitrary orders of symmetry & By periodization of a unifold design & By solving an eigen decomposition for each order \\
\hline \hline
\end{tabular}

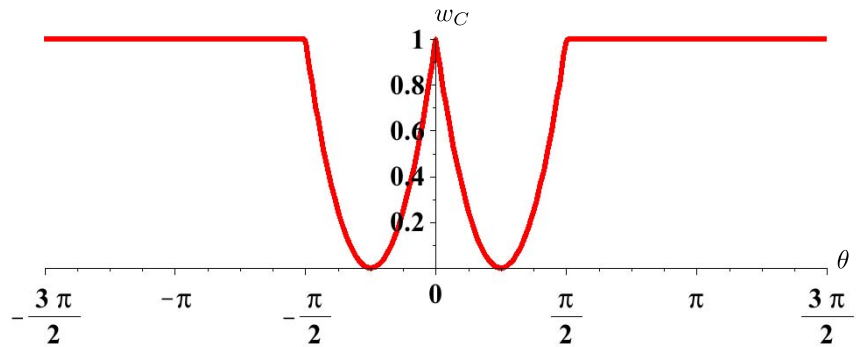

Fig. 7. Angular weighting function for the design of asymmetric wavelets (corner).
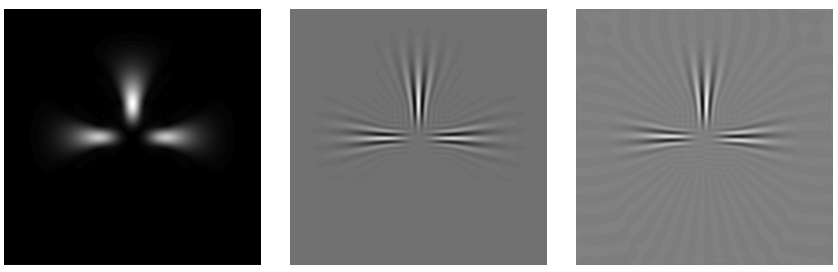

Fig. 8. T-shaped asymmetric wavelet in the space domain. From left to right: magnitude, real, and imaginary parts, respectively. Harmonics: $S=\{0, \ldots, 40\}$.
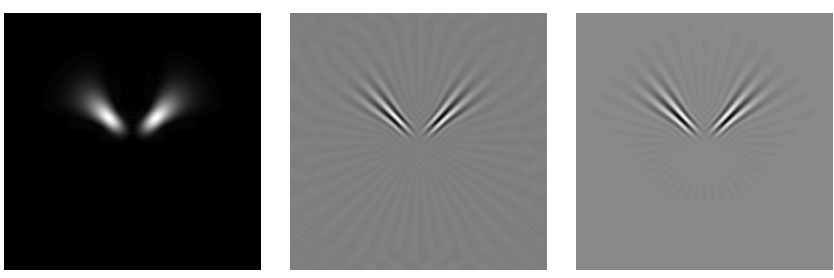

Fig. 9. Corner-shaped asymmetric wavelet in the space domain. From left to right: magnitude, real, and imaginary parts, respectively. Harmonics: $S=\{0, \ldots, 40\}$.

where $C_{C}$ is the normalization constant $(4 / \pi)^{2}$. The corresponding kernel is

$$
W_{C}(q)=\frac{\pi^{2} q^{2} \sin (\pi q)-32 \sin \left(\frac{\pi}{2} q\right)+8 \pi q\left(1+\cos \left(\frac{\pi}{2} q\right)\right)}{\pi^{3} q^{3}} .
$$

The wavelet obtained by the proposed method and its weight function can be seen in Figures 7 and 9.

\section{COMPARISON OF DifFERENT Designs}

The main features of the two designs of Section III. are summarized in Table I. We want to stress that the characteristics that address speed pertain only to the initial design of the wavelets (calculation of the design matrix $\mathbf{U}$ ). Once the wavelets have been designed, the analysis and processing of images runs at the same speed for a given number of harmonics, independent of the design.

Both approaches provide a comprehensive basis to detect symmetric junctions. The Fourier-domain design is easiest, since the radial part of the wavelet has no effect on the optimization process. The space-domain design is more cumbersome because it requires one to handle the Hankel-like integrals associated with the radial part of the wavelet. In return, it sidesteps the limitations of the Fourier domain that forbid wavelets that are asymmetric and real.

Proposition 3: No real-valued wavelets with odd symmetries can result from minimizing (27) in the Fourier domain while enforcing negative-positive pairs of harmonics.

The proof of Proposition 3 can be found in Appendix B. While the Fourier design is not appropriate when odd-symetric real wavelets are desired, this does not impair the detection of junctions as complex odd designs are still possible (using positive harmonics alone). Also, by defining the energy functional $E\{\psi\}$ in the space domain, it is possible to obtain real wavelets.

The Fourier techniques are not suitable to the design of asymmetric wavelets (e.g., corners, $\mathrm{T}$ junctions), since the Fourier design relies on the equivalence of rotational symmetries between the spatial and Fourier domains. On the contrary, the space design provides a free parametric framework.

\section{Vi. Application to Image Analysis}

Our junction- and structure-detection algorithm consists of a main part, namely, the detection of key points (i.e., centers of symmetry) followed by an optional connecting of the detected junctions, which is formulated as an optimal-pathfinding problem.

\section{A. Summary of the Algorithm}

Suppose that an $M$-fold symmetric steerable wavelet is at our disposal. Then, our algorithm to detect junctions of a given multiplicity along with the orientation of the corresponding edges, and the connections between these junctions, can be decomposed in the steps presented in Figure 10.

(1) Wavelet analysis with optimally steered wavelets: We decompose the image with the steerable wavelet. At each location and scale, the local orientation $\theta_{0}$ is determined that maximizes the "detector response" (40). The output of this first stage is a map of maximal steerable wavelet responses $Q(s, \boldsymbol{k})$ and orientations at every scale and location. 


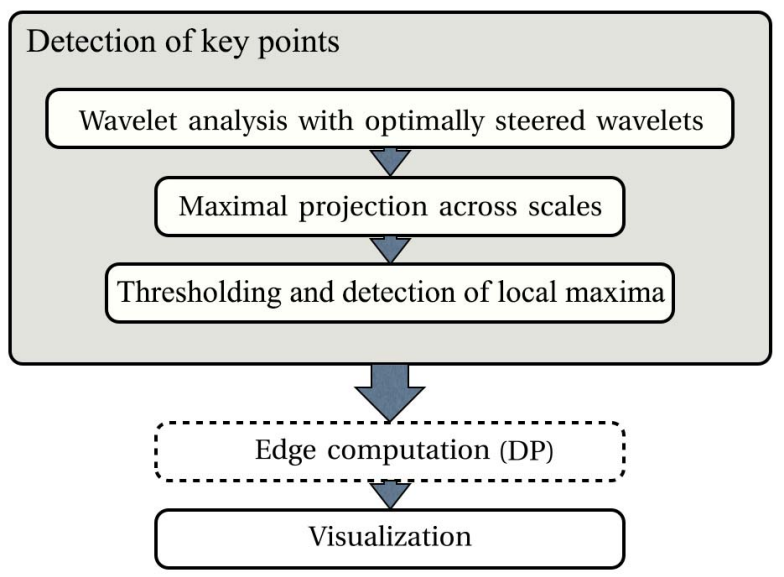

Fig. 10. The main steps of the algorithm.

(2) Maximal projection across scales: We perform a maximum-intensity projection to aggregate the key points detected at different scales. The detectors have normalized energy across scales, thus, a maximal projection across scales is a meaningful choice. For each position, we keep the coefficient corresponding to the largest detector response across existing scales, like

$$
Q(\boldsymbol{k})=\max _{s}(Q(s, \boldsymbol{k})) .
$$

(3) Thresholding and the detection of local maxima: We assume that the pixels that correspond to key points are sparse in the image. Background pixels correspond to points with a detector response smaller than the mean of the whole image. We additionally apply local nonmaximum suppression over a user-predefined window in order to prevent multiple detections of the same junction.

(4) Edge computation using dynamic programming (Optional): Based on the key points detected after Step (3), we use dynamic programming to establish paths between all possible pairs of symmetry centers that are closer than a given threshold. "True" junctions are finally extracted from the set of possible edges by favoring configurations that involve low-cost paths that are coherent with each type of center of symmetry.

The details of Steps (1) and (4) are given next.

\section{B. Steering}

In this section, we present a novel analytical method that applies efficiently to all detectors. We determine the optimal steering angle by maximizing a trigonometric polynomial in $\theta_{0}$, which can be achieved by computing the roots of its first-order derivative.

The key points in the image correspond to maxima in the response of the wavelet detector. The wavelets therefore have to be "steered" to look for the angle that elicits the largest response. Let $\left\{q_{1}, \ldots, q_{N}\right\}$ denote the coefficients of the $N$ channels computed by analyzing the input image at scale $s$ and position $\boldsymbol{k}$ using the original $N$-channel wavelets $\left\{\xi_{s, \mathbf{k}}^{\left(n_{1}\right)}(r, \theta), \ldots, \xi_{s, \mathbf{k}}^{\left(n_{N}\right)}(r, \theta)\right\}$. For the steering, we want to rotate the wavelet $\psi$ at each $(s, \boldsymbol{k})$ and find the maximum of $\left|\left\langle\psi_{s, \boldsymbol{k}}\left(\cdot, \cdot+\theta_{0}\right), f\right\rangle\right|$ as a function of $\theta_{0}$. For a given $(s, \boldsymbol{k})$, we thus rewrite the function to maximize as

$$
Q\left(s, \boldsymbol{k}, \theta_{0}\right)=\left|\left\langle\sum_{n \in S} \mathrm{e}^{\mathrm{j} n \theta_{0}} u_{n} \xi_{s, \boldsymbol{k}}^{(n)}, f\right\rangle\right|^{2}=\left|\sum_{n \in S} q_{n} \overline{u_{n}} \mathrm{e}^{-\mathrm{j} n \theta_{0}}\right|^{2},
$$

using (5) and noting that the inner product is conjugate-linear in the first argument.

We propose to maximize (40) by establishing a root-finding task on its derivative with respect to $\theta_{0}$. The order of the polynomial to solve can be reduced substantially by making a change of variable, relying on the fact that we use harmonics that are multiples of $M$ for an $M$-fold pattern. We introduce the variable $z=\mathrm{e}^{\mathrm{j} M \theta_{0}}$ for the given set of harmonics $S=\left\{M k: k=k_{0}, k_{0}+1, \ldots, k_{0}+N-1\right\}$. The polynomial to be maximized on the unit circle hence becomes

$$
Q(z)=\left(\sum_{k=k_{0}}^{k_{0}+N-1} r_{k} z^{k}\right) \overline{\left(\sum_{k=k_{0}}^{k_{0}+N-1} r_{k} z^{k}\right)}
$$

where $r_{k}=q_{M k} \overline{u_{M k}}$. Extrema of this expression are found by looking for the zeros of its derivative with respect to $\theta_{0}$. After finding the points that satisfy this condition, we evaluate $Q(z)$ at all of them to find the one corresponding to a maximum.

It is important to note that, on the unit circle, $Q$ can be rewritten as

$$
Q(z)=\sum_{k} s_{k} z^{k},
$$

where $s=r[\cdot] * \bar{r}[-\cdot]$ is the discrete auto correlation of $r$. The derivative then yields

$$
\frac{\mathrm{d}}{\mathrm{d} \theta_{0}} Q(z)=(\mathrm{j} M) \sum_{k} k s_{k} z^{k}
$$

This form greatly simplifies the processing as the complex roots of $\frac{\mathrm{d}}{\mathrm{d} \theta_{0}} Q(z)$ can be easily computed, now that an analytical expression of the coefficients of this polynomial is known.

Let $\gamma$ be the root on the unit circle for which $Q(\gamma)$ is maximal. From the definition $z=\mathrm{e}^{\mathrm{j} M \theta_{0}}$, the optimal steering angle is given by

$$
\theta_{0}=\frac{\measuredangle \gamma}{M}
$$

Due to the $M$-fold symmetry, $\theta_{0}+m \frac{2 \pi}{M}, m \in \mathbb{Z}$, are equivalent solutions.

\section{Connecting Junctions}

We propose an approach based on dynamic programming in order to link the detected centers of symmetry and segment the objects of interest [50]. The best path between two centers of symmetry is searched on a discrete grid built around axes indexed by $k$ and $u$, the former corresponding to the straight line joining the starting and end points and the latter to the perpendicular line to $k$ going through the starting point (i.e., $k=0$ ). We refer to $u_{k}$ as the value on the $u$ axis of the 
decision node at location $k$. We define the cost $c$ of the path connecting two junctions up to node $u_{k+1}$ as

$$
c\left(u_{k+1}\right)=c\left(u_{k}\right)+\frac{\lambda}{L_{\zeta}}\left(\sum_{(x, y) \in \zeta} I(x, y)\right)+(1-\lambda)\left|u_{k+1}-u_{k}\right|,
$$

where $\zeta$ is the segment that joins $u_{k}$ and $u_{k+1}, L_{\zeta}$ its length, and $I$ the input image. The cost is therefore equal to the cost up to the previous node $u_{k}$, plus two terms weighted by the problem-dependent parameter $\lambda \in[0,1]$. The first term ensures fidelity to data as it is composed of the integral on the input image over the straight line between $u_{k}$ to $u_{k+1}$. This formulation favors paths that explore dark pixels; it can be negated when bright paths are searched for. The second term enforces smoothness by penalizing large displacements along the $u$ axis. The solution found at any step $k$ is guaranteed to be optimal as it is built by propagating the optimal solution up to this step.

As we have no prior information on which other points each center of symmetry links to, we apply a global approach to connect the junctions in the whole image. For a given center, we therefore compute all possible paths linking it to its neighbors within a certain range of distance and save their cost. We proceed in this fashion for all points and therefore obtain a list of costs for each possible connection between two centers of symmetry. After ordering this list in a decreasing order with respect to the cost value, we start with the first entry, then go through each of the following and draw the valid connections. In order to determine which connections are most likely to be correct, we rely on the knowledge of the order of symmetry of each center. We define a link as valid under two conditions. First, the centers involved have to be "free": they have to be connected to less than $M$ other points if they have $M$-fold symmetry. Then, as connections in an $M$-fold center are assumed to be spaced by an angle $\frac{\pi}{M}$, the angle between the new link and the already validated ones is checked for coherency. We proceed in this way until all the centers of symmetry are connected.

\section{EXPERIMENTAL RESULTS}

Our symmetry-detection algorithm has been implemented as a plug-in for the open-source image-processing software ImageJ [51]. In our implementation, we use Simoncelli's isotropic mother wavelets [39] with dyadic scale progressions. To evaluate the performance of the algorithm, we tested it on a variety of synthetic images in the presence of noise and, finally, on real microscopic images. The aim of this section is to measure to which degree our methods honor translation, rotation, and scale invariance, as well as its robustness against noise.

We evaluate two versions of the space design: a real version with positive and negative harmonic pairs; and a complex version with positive harmonics only. We compare these results with the ones given by the Fourier template. We fix the number of harmonics for all cases: $S=\{3 n: n=0,1, \ldots, 9\}$ for the complex wavelets and $S=\{3 n: n=-8,-7, \ldots, 7,8\}$ for the real wavelets.
In [52], Sage et al. showed experimentally that the spectral power density of fluorescence-microscopy images is isotropic and is well represented by the power law $\frac{1}{\|\omega\|^{s}}$, where $\omega$ is the radial spatial frequency, and $s$ is the fractal exponent. The corresponding fractional Brownian-motion model [53] is typical of a broad category of natural images [54]. To generate our test images, we assume that we can represent the background signal (autofluorescence) present in biological images with the given model. We consider the background signal as noise to the detection problem.

In general, to make a quantitative evaluation, we compute the Jaccard index, and both position and angle root meansquare errors. The Jaccard index is typically used for comparing the similarity and diversity of finite sample sets. In our case, it concerns the set of the ground truth values $A$ and the set of the detections $B$. The Jaccard index is defined as

$$
J(A, B)=\frac{|A \cap B|}{|A \cup B|},
$$

in words, the size of the intersection divided by the size of the union of the two sets. If $J(A, B)=1$, we have perfect detections. If $J(A, B)=0$, all of our detections are false positive. Otherwise, the Jaccard index takes values $0<J(A, B)<1$, depending on the number of false positive and negative results. We illustrate the Jaccard as percentage, ranging from 0 to 100 .

\section{A. Robustness Against Noise}

First, we generate a series of $(1024 \times 1024)$ test images, where we control the location of the junctions and the angles. To separate distinct effects, in this experiment we use only the first wavelet scale to make detections. We then corrupt the images with isotropic Brownian motion, with a mean of zero and a standard deviation of $1,2, \ldots, 100$. We intend to detect 643 junctions, with 2 different angle values: 0 and 180 degrees.

To make a quantitative evaluation we compute the Jaccard index, and both position and angle root mean-square errors. In the evaluation phase, we use the Hungarian algorithm to match the detections with the nodes of the original grid. The detections are accepted if they are closer than 5 pixel from the original nodes. Otherwise, they are counted as false positives. The RMSE error is computed for the matched detections.

An illustration of the results (crop of one of the test images) can be seen in Figure 11. As visible from these images, our algorithm performs much better than structure tensor and Hessian-based methods in case of strong noise. The Jaccard index is presented in Figure 12, and the RMS errors in Figure 13.

Figure 11 also gives an intuitive illustration of the results of the Jaccard index and the RMS errors. We can see that the noise level of $\sigma=30$, which corresponds to the test image Figure $11 \mathrm{~A}$, is in the range when our method performs the best (Jaccard index 100\%, negligible RMS errors). The noise level of $\sigma=80$, which corresponds to the test image Figure $11 \mathrm{~B}$ corresponds to the point where the Jaccard index curve start to cut off from $100 \%$.

Based on the graphs, we can say that the Fourier and the complex spatial design performed best. The real spatial design 

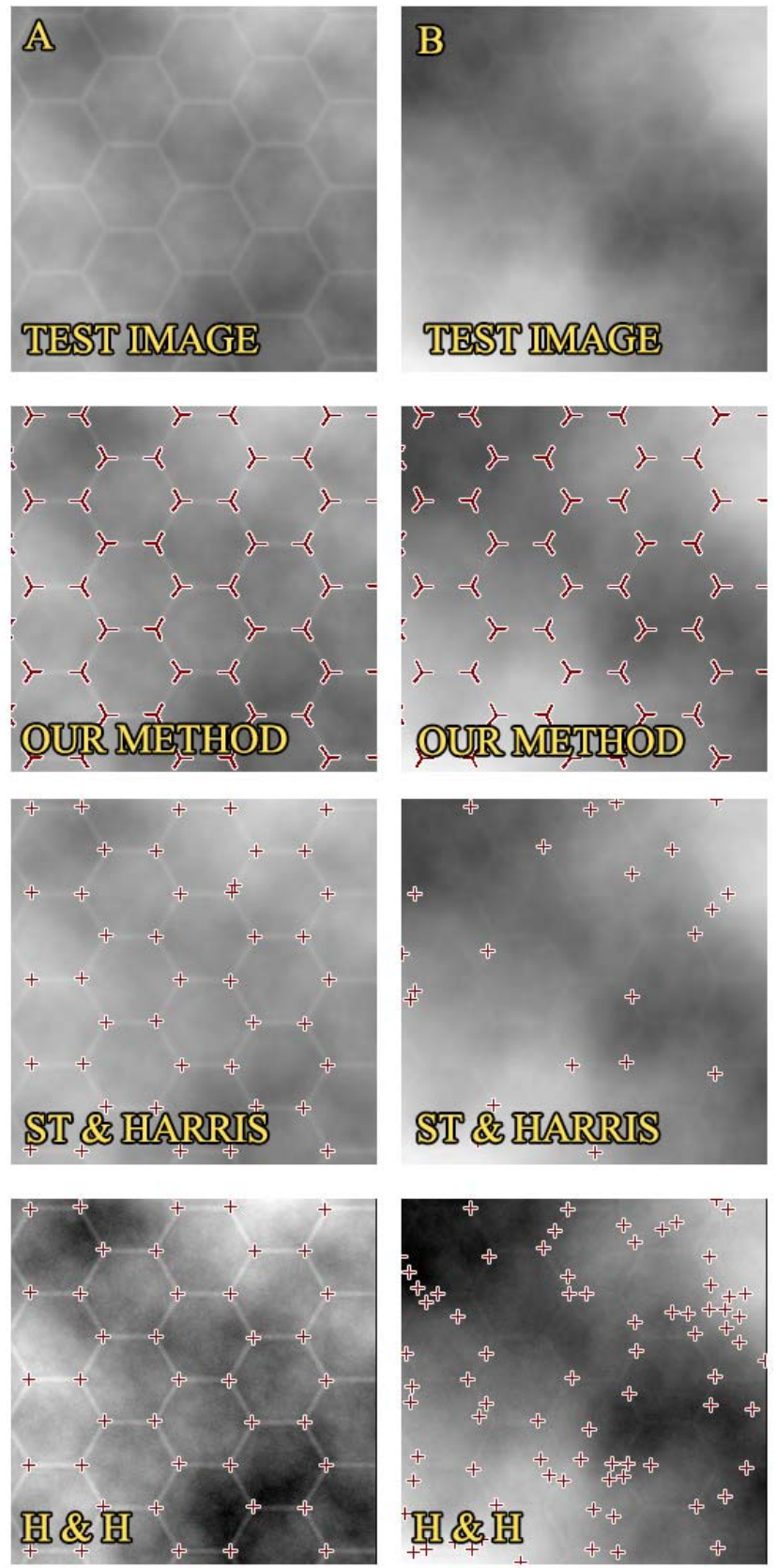

Fig. 11. From left to right and top to bottom: test image corrupted with isotropic Brownian motion (mean 0, standard deviation 30) and (mean 0, standard deviation 80); detections with our method on image A, and B; detections with Harris' corner detector based on the structure tensor on image A, and B; detections with Harris' corner detector based on the largest eigenvalues of the Hessian on Image A, and B. To generate the results we have used the ImageJ/Fiji built-in Hessian plug-in. To provide a fair comparison, we used only the first wavelet scale of our method to make detections.

had difficulties under heavy noise; they produced a bias even in the noise-free case. This has two explanations. First, the shape of the wavelet (Figure 1) contains oscillations related to the Hankel functions. When matching, the algorithm tries to fit the junction with the oscillations, such that the maxima overlap. Second, when generating the wavelet we had to use more harmonics (17 channels in total) to achieve the same blade size, hence, the same detection range. When steering the wavelets, these extra harmonics mean that we have to find

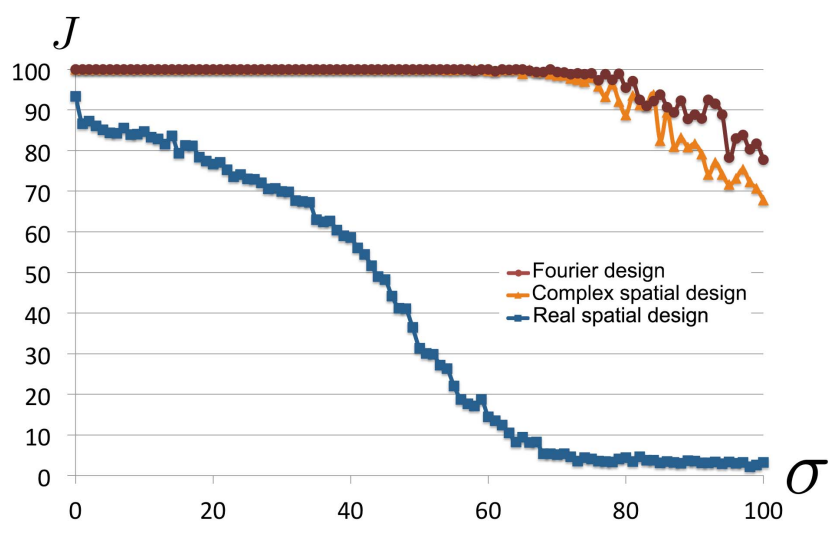

Fig. 12. Jaccard index $J$ under isotropic Brownian motion, as a function of the standard deviation $\sigma$ of the noise.
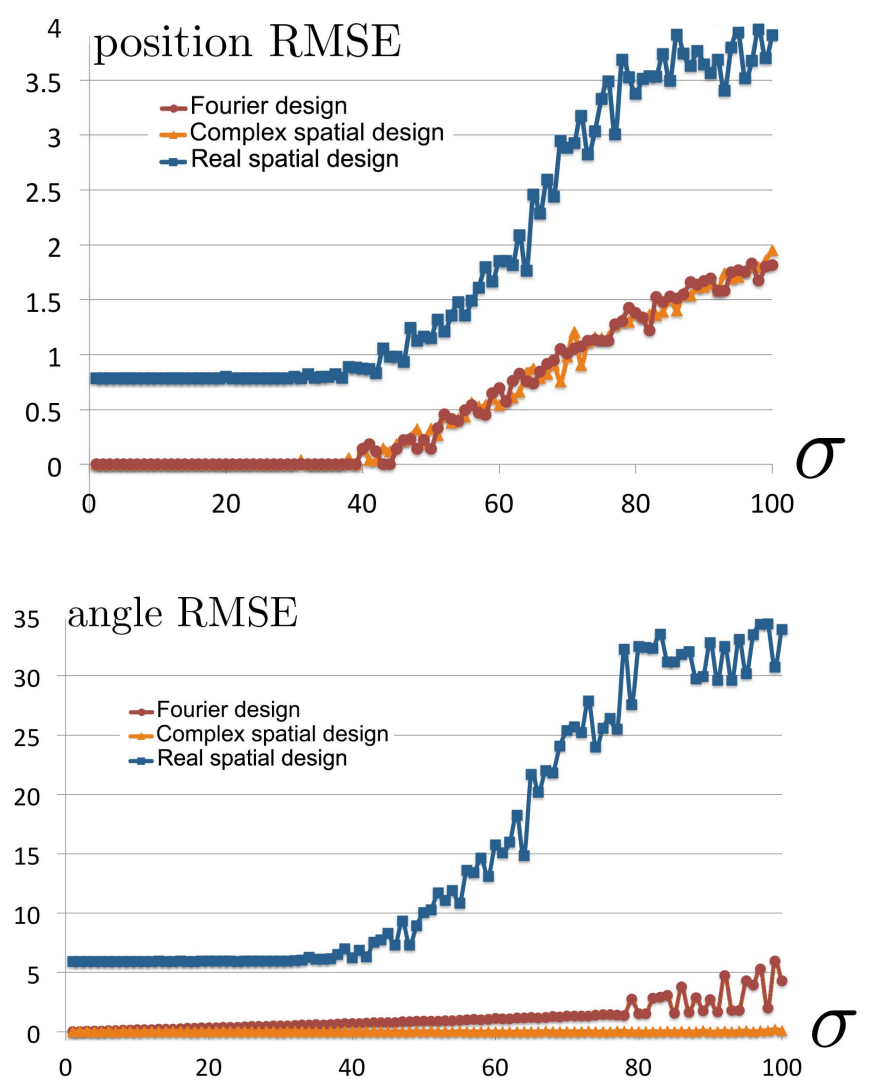

Fig. 13. Position and angle error in the root-mean-square (RMSE) sense under isotropic Brownian motion, as a function of the standard deviation $\sigma$ of the noise.

the roots of a polynomial of a much higher order (17 instead of 10), thus increasing numerical errors.

\section{B. Rotation Invariance}

The rotation invariance property of our method is illustrated in Figure 14. The junctions were rotated with 10 degree steps with respect to their previous state. The centers of the junctions were correctly detected in all cases. The number next to the junctions correspond to the detected rotation angle of the junctions with respect to their original state. 

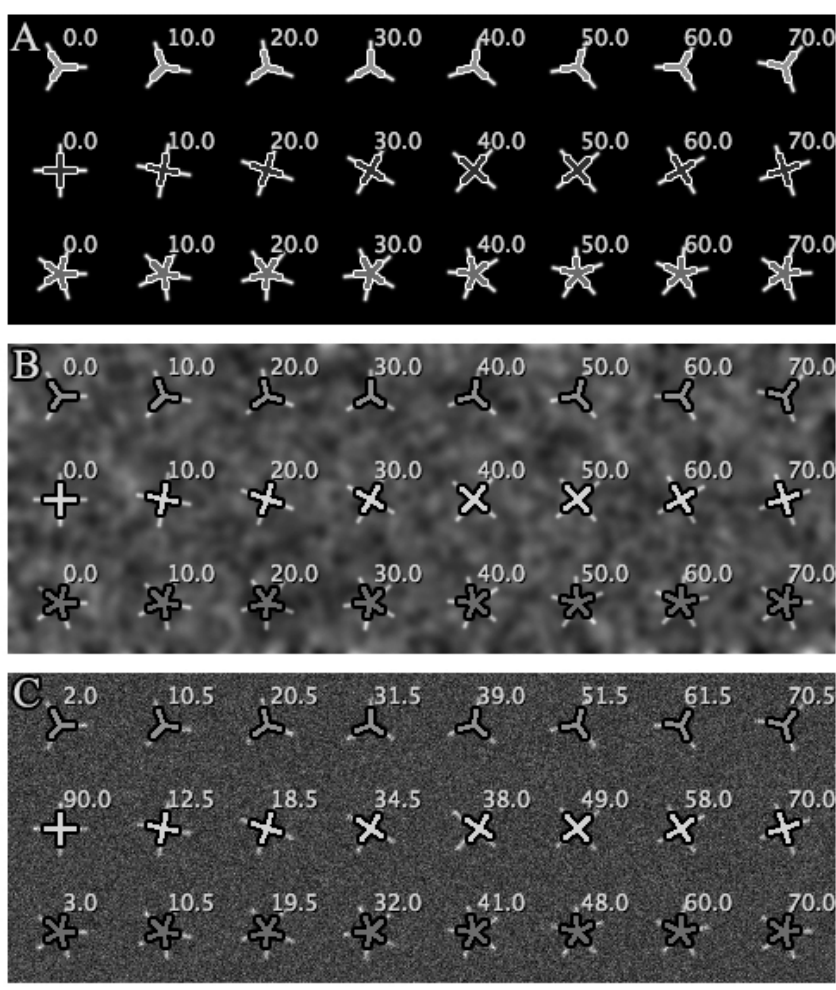

Fig. 14. Illustration of the rotation invariance of our method. From top to bottom: A: original test image, B: test image with additional Brownian motion, C: test image with additional Gaussian noise. The junctions were rotated with 10 degree steps. The centers of the junctions were correctly detected in all cases. The number next to the junctions correspond to the detected rotation angle of the junctions with respect to their original state.

We generated another series of $(1024 \times 1024)$ test images. First, we built a synthetic grid as in the previous experiments, and then rotated it around its center with steps of 1 degree, from 0 to 360 degree. We finally applied isotropic Brownian motion of zero mean and standard deviation 30 on these images. This amount of noise corresponds to the one illustrated in Figure 11 A. As in Section VII. A, we used only the first wavelet scale to make detections, and we fixed the window size to $30 \times 30$ pixel. We intend to detect approximately 640 junctions (some nodes may "fall out" of the image when rotated) with specific angle values.

To make a quantitative evaluation, we computed the Jaccard index (Figure 15), and the RMS error for position and angle (Figure 16). The graphs confirm that our method is essentially rotation-invariant and performs well, independently of the orientation of the junctions.

\section{Multiscale Properties}

We present results challenging the multiscale aspect of our approach using the test image shown in Figure 17. This synthetic image shows a uniform tiling of the hyperbolic plane and exhibits three-, and four-fold local symmetries of various size. The goal was to identify these junctions at different scales.

We generate yet another series of $(1024 \times 1024)$ test images. First, we built a synthetic grid as in the experiments of

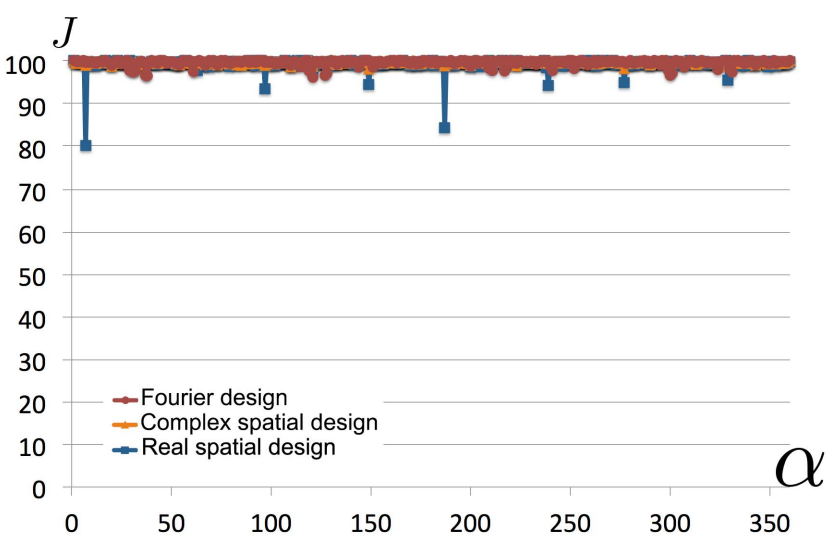

Fig. 15. Jaccard index $(J)$ under isotropic Brownian motion of zero mean and standard deviation 30 for rotations $\alpha$ from 0 to 360 degree.

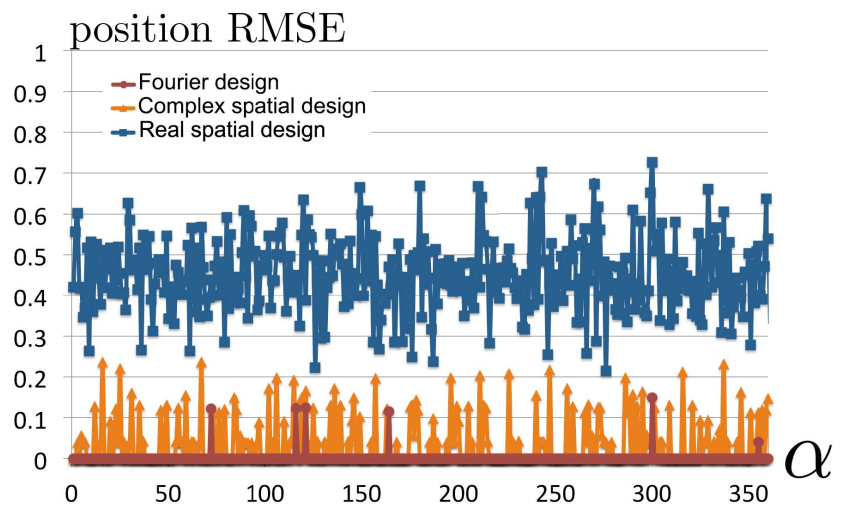

angle RMSE

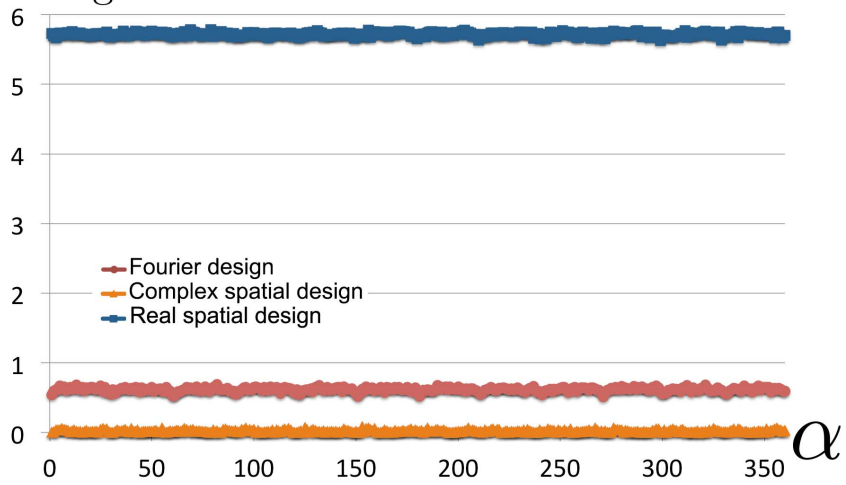

Fig. 16. Position and angle error in the sense of root mean square under isotropic Brownian motion of zero mean and standard deviation 30 for rotations $\alpha$ from 0 to 360 degree.

Sections VII A-B, and then changed the profile of the edges from thickness 1 to 5.1 with steps of 0.1 (see Figure 18). We added isotropic Brownian motion of zero mean and standard deviation 10 . We intend to detect approximately 85 junctions with specific angle values. We placed the nodes further away than before, such that they don't interfere with each other when the thickness increases. In this way, we can clearly observe the effect of different scales.

To make a quantitative evaluation, we computed the RMS error for position and angle (as seen in Figure 16). We use 


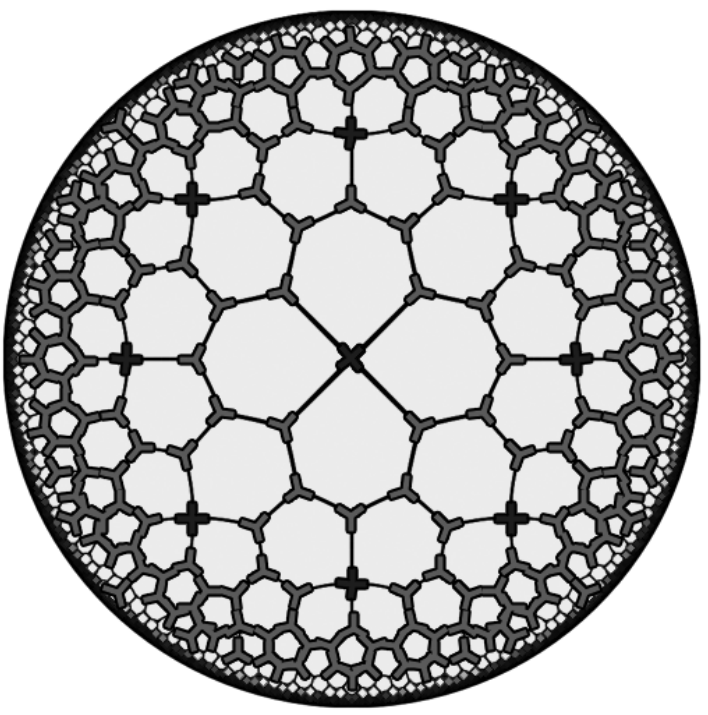

Fig. 17. Detection results for synthetic data: uniform tiling of the hyperbolic plane exhibiting three-, and four-fold local symmetries $(512 \times 512$ pixels $)$. Three-fold detections are highlighted in light grey, four-fold ones in dark grey.
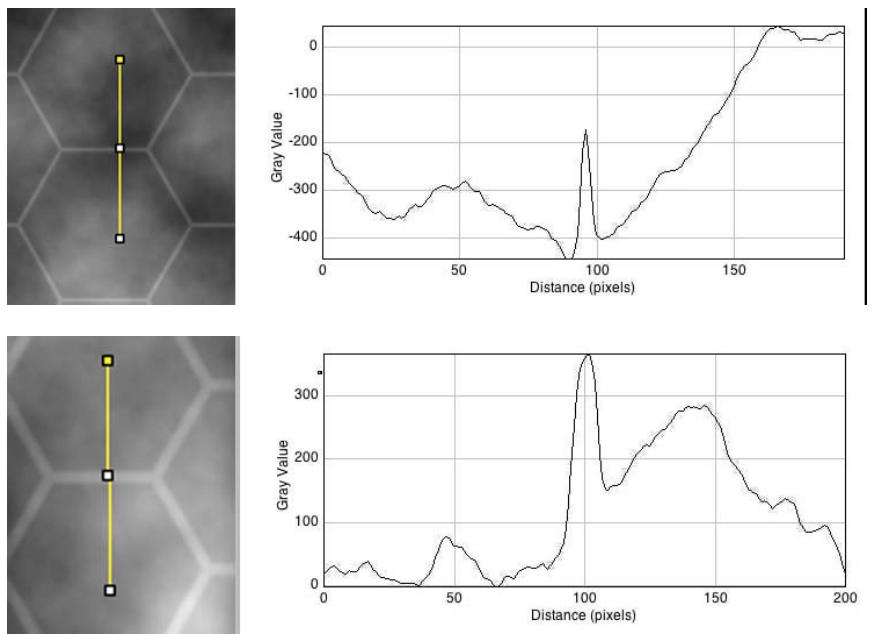

Fig. 18. Test images of different line thickness, corrupted with isotropic Brownian motion (mean 0, standard deviation 10). From top to bottom: line thickness 1.0 and 5.0, respectively.

the Jaccard index to decide whether to make the decisions at higher scale or not: when the Jaccard index is getting lower, we jump to the next scale.

Based on Figure 19, we claim that our method is essentially scale-invariant and performs well, independently of the scale of the pattern.

\section{Practical Applications}

Figure 20 features a microscopic view of a honeycomb. This biological structure is composed of a mass of cells that naturally tend to exhibit a close-to-perfect hexagonal structure. The detection results of our method are shown on the right. They are accurate, both concerning the detection of junctions and their linking, which was expected from the regularity of the structure.
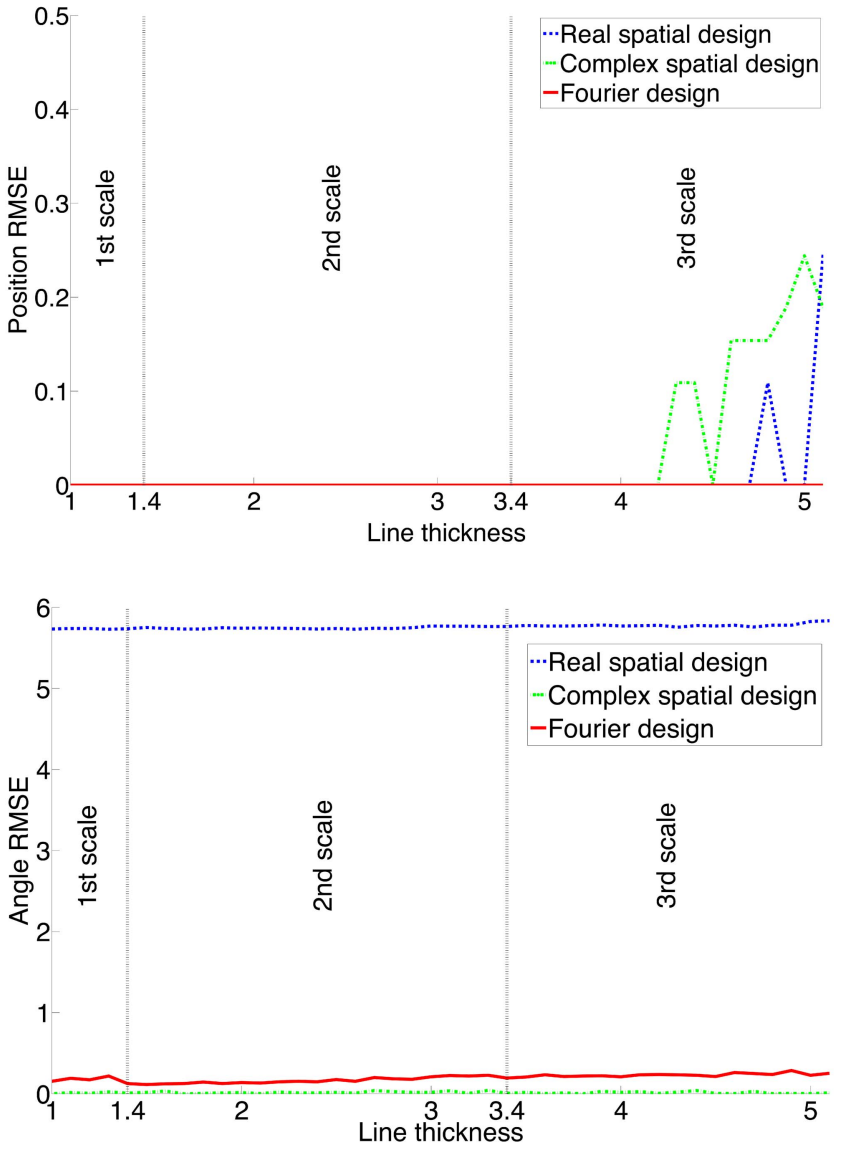

Fig. 19. Position and angle error in the sense of root mean square under isotropic Brownian motion of zero mean and standard deviation 10 for scaling from 1 to 5.1 .
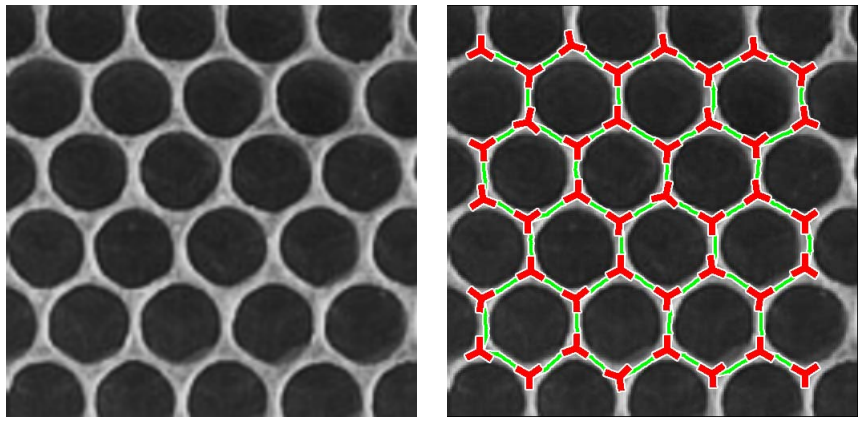

Fig. 20. Detection of three-fold junctions and segmentation in the brightfield microscopic image of a honeycomb. Left: original $(256 \times 256)$ image. Right: detection results using the proposed approach.

The image of Figure 21 contains hexagonal embryonic stem cells imaged by fluorescence microscopy with three-foldsymmetric junctions. The detection task is more challenging than in the honeycomb image due to the variety of orientations and sizes of the junctions. In this case, the method yielded again good results, which suggests that our algorithm works well even if the structure are only semi-regular and corrupted by Poisson (photon counting) noise.

Figure 22 features a picture of endothelial cells of the cornea with the detection results. 

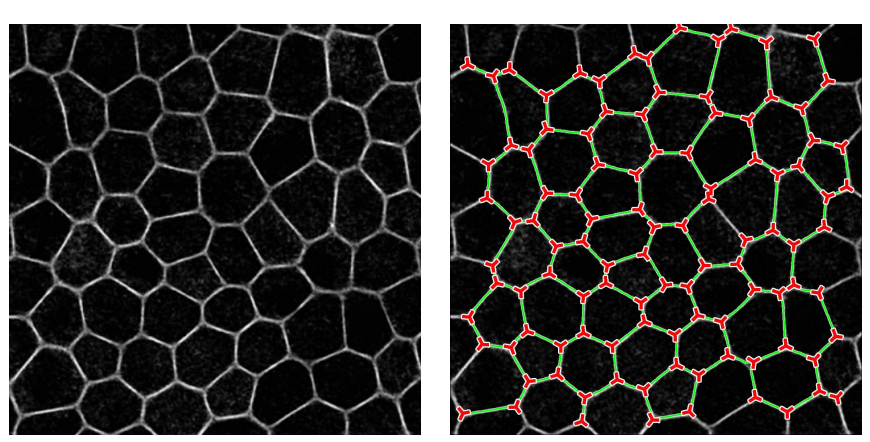

Fig. 21. Detection and linking of three-fold junctions in a fluorescence microscopic image of embryonic stem cells. Left: original $(512 \times 512)$ image. Right: detection and linking result using the proposed approach.

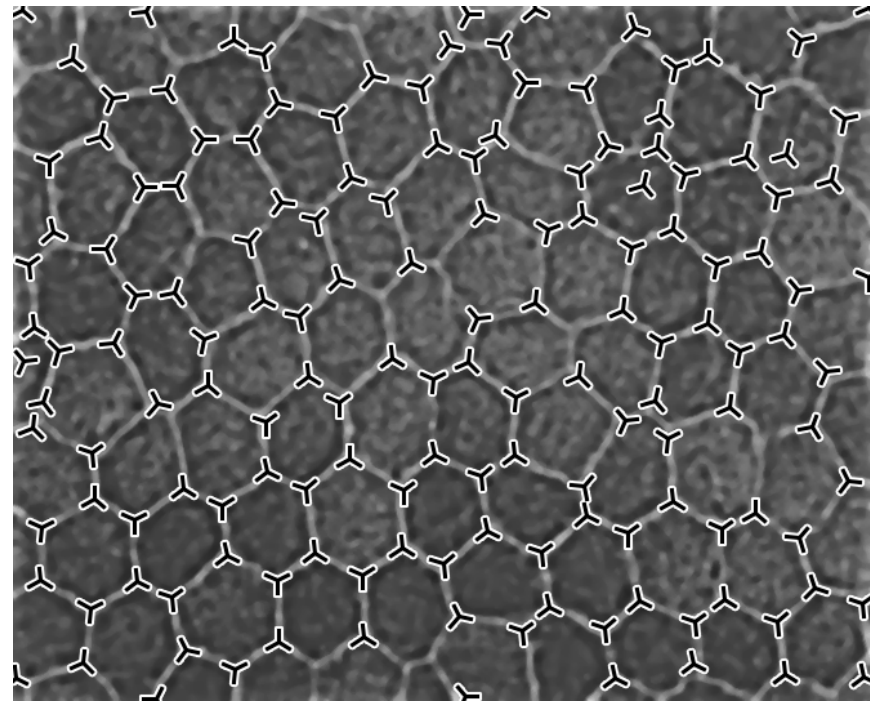

Fig. 22. Detection of three-fold junctions in a picture of endothelial cells of the cornea. Image courtesy of Fischbarg [55].

\section{CONCLUSION}

In this paper, we presented a general wavelet-based framework to detect of symmetric junctions in images. Our approach is multiscale, rotation-invariant, robust to noise, and can be tuned to identify arbitrary symmetries. It is hence possible to obtain a precise estimate of the location of junctions across a large range of scales and at any orientation. We proposed an algorithm that combines detectors of local symmetry and dynamic programming to handle images featuring multifold junctions. The effectiveness of our approach in practical applications was demonstrated on synthetic and real biology data in the presence and absence of noise.

\section{APPENDIX}

\section{A. Proof of Proposition 2}

$$
\begin{aligned}
\xi^{(n)} & (r, \theta) \\
& =\mathscr{F}^{-1}\left\{\hat{h}(\rho) \mathrm{e}^{\mathrm{j} n \varphi}\right\}(r, \theta) \\
& =\frac{1}{(2 \pi)^{2}} \int_{0}^{\infty} \int_{-\pi}^{\pi} \mathrm{e}^{\mathrm{j} r \rho \cos (\varphi-\theta)} \hat{h}(\rho) \mathrm{e}^{\mathrm{j} n \varphi} \mathrm{d} \varphi \rho \mathrm{d} \rho \\
& =\frac{1}{(2 \pi)^{2}} \mathrm{e}^{\mathrm{j} n \theta} \mathrm{e}^{-\mathrm{j} n \frac{\pi}{2}} \int_{0}^{\infty} \rho \hat{h}(\rho) \int_{-\pi}^{\pi} \mathrm{e}^{\mathrm{j}(n \alpha+r \rho \sin \alpha)} \mathrm{d} \varphi \mathrm{d} \rho
\end{aligned}
$$

$$
=\frac{\mathrm{j}^{n}}{2 \pi} \mathrm{e}^{\mathrm{j} n \theta} \int_{0}^{\infty} \rho \hat{h}(\rho) \mathrm{J}_{n}(r \rho) \mathrm{d} \rho,
$$

where we have made the change of variable $\alpha=\varphi-\theta+\frac{\pi}{2}$. We used the integral representation of the $n$ th-order Bessel function of the first kind. For integer values of $n$, it is

$$
\mathrm{J}_{n}(y)=\frac{1}{2 \pi} \int_{-\pi}^{\pi} \mathrm{e}^{-\mathrm{j}(n \tau-y \sin \tau)} \mathrm{d} \tau .
$$

We also note that the direct link between the Bessel functions of negative and positive integer orders is given by

$$
\mathrm{J}_{-n}(y)=(-1)^{n} \mathbf{J}_{n}(y) \text {. }
$$

In addition, since $\boldsymbol{x}=(r \cos \theta, r \sin \theta)$ and $\boldsymbol{\omega}=(\rho \cos \varphi, \rho \sin \varphi),\langle\boldsymbol{x}, \boldsymbol{\omega}\rangle=r \rho \cos (\theta-\varphi)=$ $r \rho \cos (\varphi-\theta)$.

\section{A. Proof of Proposition 3}

The quadratic Fourier-domain energy term to minimize takes the form

$$
\begin{aligned}
E\{\hat{\psi}\} & \frac{1}{2 \pi} \int_{-\pi}^{\pi}|\hat{\psi}(\varphi)|^{2} w(\varphi) \mathrm{d} \varphi \\
= & \frac{1}{2 \pi} \int_{-\pi}^{\pi}\left(\sum_{n=-N}^{N} u_{n} \mathrm{e}^{\mathrm{j} n \varphi}\right)\left(\sum_{n^{\prime}=-N}^{N} u_{n^{\prime}} \mathrm{e}^{\mathrm{j} n^{\prime} \varphi}\right) w(\varphi) \mathrm{d} \varphi \\
= & \sum_{n=-N}^{N} \sum_{n^{\prime}=-N}^{N} u_{n} \bar{u}_{n^{\prime}} \frac{1}{2 \pi} \int_{-\pi}^{\pi} \mathrm{e}^{\mathrm{j}\left(n-n^{\prime}\right) \varphi} w(\varphi) \mathrm{d} \varphi \\
= & \frac{1}{2} \sum_{n=-N}^{N} \sum_{n^{\prime}=-N}^{N} u_{n} \bar{u}_{n^{\prime}} \frac{1}{2 \pi} \\
& \times \int_{-\pi}^{\pi}\left(\mathrm{e}^{\mathrm{j}\left(n-n^{\prime}\right) \varphi} w(\varphi)+\mathrm{e}^{\mathrm{j}\left(n^{\prime}-n\right) \varphi} w(-\varphi)\right) \mathrm{d} \varphi \\
= & \frac{1}{2} \sum_{n=-N}^{N} \sum_{n^{\prime}=-N}^{N} u_{n} \bar{u}_{n^{\prime}} W\left(n-n^{\prime}\right) \\
& \left.+\sum_{n=-N}^{N} \sum_{n^{\prime}=-N}^{N} u_{n} \bar{u}_{n^{\prime}} W\left(n^{\prime}-n\right)\right)
\end{aligned}
$$

where we have made the assumption that $w$ is even, with $w(\varphi)=w(-\varphi)$.

The Fourier coefficients of $w$ are computed as

$$
W\left(n-n^{\prime}\right)=\frac{1}{2 \pi} \int_{-\pi}^{\pi} \mathrm{e}^{\mathrm{j}\left(n-n^{\prime}\right) \theta} w(\theta) \mathrm{d} \theta .
$$

These calculations show that the eigenvalue problem involve a real and symmetric Toeplitz matrix $\mathbf{W}$, which necessarily has real eigenvectors. In addition, the eigenvectors of a symmetric Toeplitz matrix are either symmetric or anti-symmetric around their center [56].

1) Symmetric case: $\hat{\psi}$ can be written as a sum of cosines with purely real coefficients like

$$
\sum_{n=-N}^{N} u_{n} \mathrm{e}^{\mathrm{j} n \varphi}=u_{0}+\sum_{n=1}^{N} 2 u_{n} \cos (n \varphi) .
$$


Thus, $\hat{\psi}$ is purely real, so $\psi$ is Hermitian-symmetric. As a result, $|\psi|$ is symmetric with respect to the origin.

2) Anti-symmetric case: $\hat{\psi}$ can be written as a sum of sines with purely imaginary coefficients like

$$
\sum_{n=-N}^{N} u_{n} \mathrm{e}^{\mathrm{j} n \varphi}=\sum_{n=1}^{N} 2 \mathrm{j} u_{n} \sin (n \varphi) .
$$

Thus, $\hat{\psi}$ is purely imaginary, so $\psi$ is Hermitianantisymmetric. As a result, $|\psi|$ is symmetric with respect to the origin.

In conclusion, $|\psi|$ can have only even orders of symmetry. Now, we shall attempt to observe how this property influences the detection of odd symmetries. We make the natural assumption that the image $f$ is real, so that

$$
|\langle\psi, f\rangle|^{2}=(\langle\operatorname{Re}(\psi), f\rangle)^{2}+(\langle\operatorname{Im}(\psi), f\rangle)^{2} .
$$

If we rotate $\psi$ by $\pi$, the magnitude of the wavelet coefficients doesn't change (only the sign of $\langle\operatorname{Re}(\psi), f\rangle$ or $\langle\operatorname{Im}(\psi), f\rangle$ can be different). It means that the magnitude of the wavelet coefficients cannot distinguish a pattern from the rotation the of same pattern by $\pi$. Thus, it is not suitable for detecting odd symmetries.

We note that the same derivation is valid if the harmonics are multiples of some integer $\kappa$.

\section{REFERENCES}

[1] Z. Püspöki, C. Vonesch, and M. Unser, "Detection of symmetric junctions in biological images using 2D steerable wavelet transforms," in Proc. 10th IEEE Int. Symp. Biomed. Imag., Nano Macro (ISBI), San Francisco CA, USA, Apr. 2013, pp. 1496-1499.

[2] F. Osakada et al., "Toward the generation of rod and cone photoreceptors from mouse, monkey and human embryonic stem cells," Nature Biotechnol., vol. 26, no. 2, pp. 215-224, Feb. 2008.

[3] F. Zhao, P. R. S. Mendonca, R. Bhotika, and J. V. Miller, "Model-based junction detection algorithm with applications to lung nodule detection," in Proc. 4th IEEE Int. Symp. Biomed. Imag., Nano Macro (ISBI), Arlington, VA, USA, Apr. 2007, pp. 504-507.

[4] G.-S. Xia, J. Delon, and Y. Gousseau, "Accurate junction detection and characterization in natural images," Int. J. Comput. Vis., vol. 106, no. 1, pp. 31-56, Jan. 2014.

[5] M. Maire, P. Arbelaez, C. Fowlkes, and J. Malik, "Using contours to detect and localize junctions in natural images," in Proc. IEEE Comput. Soc. Conf. Comput. Vis. Pattern Recognit. (CVPR), Anchorage, AK, USA, Jun. 2008, pp. 1-8.

[6] T.-A. Pham, M. Delalandre, S. Barrat, and J.-Y. Ramel, "Accurate junction detection and reconstruction in line-drawing images," in Proc. 21st Int. Conf. Pattern Recognit. (ICPR), Tsukuba, Japan, Nov. 2012, pp. 693-696.

[7] W. Liang and H. Yunan, "Vision-aided navigation for aircrafts based on road junction detection," in Proc. IEEE Int. Conf. Intell. Comput. Intell. Syst. (ICIS), vol. 4. Shanghai, China, Nov. 2009, pp. 164-169.

[8] S. Tsogkas and I. Kokkinos, "Learning-based symmetry detection in natural images," in Computer Vision-ECCV (Lecture Notes in Computer Science), vol. 7578, A. Fitzgibbon, S. Lazebnik, P. Perona, Y. Sato, and C. Schmid, Eds. Florence, Italy: Springer, 2012, pp. 41-54.

[9] T.-A. Pham, M. Delalandre, S. Barrat, and J. Ramel, "A robust approach for local interest point detection in line-drawing images," in Proc. 10th IAPR Int. Workshop Document Anal. Syst. (DAS), Gold Coast, QLD, Australia, Mar. 2012, pp. 79-84.

[10] U. Köthe, "Edge and junction detection with an improved structure tensor," in Pattern Recognition (Lecture Notes in Computer Science), vol. 2781, B. Michaelis and G. Krell, Eds. Berlin, Germany: Springer, 2003, pp. 25-32.

[11] J. Bigun, T. Bigun, and K. Nilsson, "Recognition by symmetry derivatives and the generalized structure tensor," IEEE Trans. Pattern Anal. Mach. Intell., vol. 26, no. 12, pp. 1590-1605, Dec. 2004.
[12] L. Parida, D. Geiger, and R. Hummel, "Junctions: Detection, classification, and reconstruction," IEEE Trans. Pattern Anal. Mach. Intell. vol. 20, no. 7, pp. 687-698, Jul. 1998.

[13] F. Escolano Ruiz, P. Suau Pérez, and B. Bonev, Information Theory in Computer Vision and Pattern Recognition. London, U.K.: SpringerVerlag, 2009.

[14] W. T. Freeman, "Steerable filters and local analysis of image structure," Ph.D. dissertation, School Archit. Planning, Massachusetts Inst. Technol., Cambridge, MA, USA, 1992.

[15] E. P. Simoncelli and H. Farid, "Steerable wedge filters for local orientation analysis," IEEE Trans. Image Process., vol. 5, no. 9, pp. 1377-1382, Sep. 1996.

[16] G. González, F. Aguet, F. Fleuret, M. Unser, and P. Fua, "Steerable features for statistical 3D dendrite detection," in Proc. 12th Int. Conf Med. Image Comput. Comput.-Assist. Intervent. (MICCAI), vol. 5762. London, U.K., Sep. 2009, pp. 625-632.

[17] M. Müehlich, D. Friedrich, and T. Aach, "Design and implementation of multisteerable matched filters," IEEE Trans. Pattern Anal. Mach. Intell., vol. 34, no. 2, pp. 279-291, Feb. 2012.

[18] C. Sun and P. Vallotton, "Fast linear feature detection using multiple directional non-maximum suppression," J. Microscopy, vol. 234, no. 2 , pp. 147-157, May 2009.

[19] J. Canny, "A computational approach to edge detection," IEEE Trans. Pattern Anal. Mach. Intell., vol. PAMI-8, no. 6, pp. 679-698, Nov. 1986.

[20] W. Förstner, "A feature based correspondence algorithm for image matching," Int. Arch. Photogramm. Remote Sens., vol. 26, no. 3, pp. 150-166, Aug. 1986.

[21] C. Harris and M. Stephens, "A combined corner and edge detector," in Proc. 4th Alvey Vis. Conf., Manchester, U.K., 1988, pp. 147-151.

[22] S. Mallat, A Wavelet Tour of Signal Processing: The Sparse Way. San Diego, CA, USA: Academic, 2008.

[23] T. S. Lee, "Image representation using 2D Gabor wavelets," IEEE Trans. Pattern Anal. Mach. Intell., vol. 18, no. 10, pp. 959-971, Oct. 1996.

[24] J. G. Daugman, "Complete discrete 2D Gabor transforms by neural networks for image analysis and compression," IEEE Trans. Acoust., Speech Signal Process., vol. 36, no. 7, pp. 1169-1179, Jul. 1988.

[25] J.-P. Antoine, R. Murenzi, and P. Vandergheynst, "Directional wavelets revisited: Cauchy wavelets and symmetry detection in patterns," Appl. Comput. Harmon. Anal., vol. 6, no. 3, pp. 314-345, May 1999.

[26] P. Kovesi, "Symmetry and asymmetry from local phase," in Proc. 10th Austral. Joint Conf. Artif. Intell., Perth, WA, Australia, Nov. 1997, pp. 2-4.

[27] E. J. Candès and D. L. Donoho, "New tight frames of curvelets and optimal representations of objects with piecewise $C^{2}$ singularities," Commun. Pure Appl. Math., vol. 57, no. 2, pp. 219-266, Feb. 2004.

[28] D. Labate, W.-Q. Lim, G. Kutyniok, and G. Weiss, "Sparse multidimensional representation using shearlets," Proc. SPIE, vol. 5914, p. 59140U, Sep. 2005.

[29] S. Yi, D. Labate, G. R. Easley, and H. Krim, "A shearlet approach to edge analysis and detection," IEEE Trans. Image Process., vol. 18, no. 5, pp. 929-941, May 2009.

[30] M. N. Do and M. Vetterli, "The contourlet transform: An efficient directional multiresolution image representation," IEEE Trans. Image Process., vol. 14, no. 12, pp. 2091-2106, Dec. 2005.

[31] L. Semler and L. Dettori, "Curvelet-based texture classification of tissues in computed tomography," in Proc. 13th IEEE Int. Conf. Image Process. (ICIP), Atlanta, GA, USA, Oct. 2006, pp. 2165-2168.

[32] G. Kutyniok, "Data separation by sparse representations," in Compressed Sensing: Theory and Applications. Cambridge, U.K.: Cambridge Univ. Press, 2012.

[33] S. T. Ali, J.-P. Antoine, and J.-P. Gazeau, "Multidimensional wavelets and generalizations," in Coherent States, Wavelets, and Their Generalizations (Theoretical and Mathematical Physics). New York, NY, USA: Springer, 2014, pp. 411-456. [Online]. Available: http://dx.doi.org/10.1007/978-1-4614-8535-3_14

[34] L. Jacques, L. Duval, C. Chaux, and G. Peyré, "A panorama on multiscale geometric representations, intertwining spatial, directional and frequency selectivity," Signal Process., vol. 91, no. 12, pp. 2699-2730, Dec. 2011.

[35] W. T. Freeman and E. H. Adelson, "Steerable filters for early vision, image analysis, and wavelet decomposition," in Proc. 3rd Int. Conf. Comput. Vis.. Osaka, Japan, Dec. 1990, pp. 406-415. 
[36] P. Perona, "Steerable-scalable kernels for edge detection and junction analysis," Image Vis. Comput., vol. 10, no. 10, pp. 663-672, Dec. 1992.

[37] M. Unser and N. Chenouard, "A unifying parametric framework for 2D steerable wavelet transforms," SIAM J. Imag. Sci., vol. 6, no. 1, pp. 102-135, 2013.

[38] J. P. Ward and M. Unser, "Harmonic singular integrals and steerable wavelets in $\mathrm{L}_{2}\left(\mathrm{R}^{\mathrm{d}}\right)$, , Appl. Comput. Harmon. Anal., vol. 36, no. 2, pp. 183-197, Mar. 2014.

[39] J. Portilla and E. P. Simoncelli, "A parametric texture model based on joint statistics of complex wavelet coefficients," Int. J. Comput. Vis., vol. 40 , no. 1 , pp. $49-70$, Oct. 2000.

[40] G. Jacovitti and A. Neri, "Multiresolution circular harmonic decomposition," IEEE Trans. Signal Process., vol. 48, no. 11, pp. 3242-3247, Nov. 2000.

[41] U. Köthe, "Low-level feature detection using the boundary tensor," in Visualization and Processing of Tensor Fields (Mathematics and Visualization), J. Weickert and H. Hagen, Eds. Berlin, Germany: Springer, 2006, pp. 63-79.

[42] M. Jacob and M. Unser, "Design of steerable filters for feature detection using Canny-like criteria," IEEE Trans. Pattern Anal. Mach. Intell., vol. 26, no. 8, pp. 1007-1019, Aug. 2004

[43] M. Felsberg and G. Sommer, "The monogenic signal," IEEE Trans. Signal Process., vol. 49, no. 12, pp. 3136-3144, Dec. 2001

[44] M. Unser and D. Van De Ville, "Wavelet steerability and the higherorder Riesz transform," IEEE Trans. Image Process., vol. 19, no. 3, pp. 636-652, Mar. 2010.

[45] A. Depeursinge, A. Foncubierta-Rodriguez, D. Van De Ville, and H. Müller, "Rotation-covariant texture learning using steerable Riesz wavelets," IEEE Trans. Image Process., vol. 23, no. 2, pp. 898-908, Feb. 2014

[46] M. Reisert and H. Burkhardt, "Harmonic filters for generic feature detection in 3D" in Proc. 31st Annu. Symp. German Assoc. Pattern Recognit. (DAGM), vol. 5748. Sep. 2009, pp. 131-140.

[47] H. Skibbe, Q. Wang, O. Ronneberger, H. Burkhardt, and M. Reisert, "Fast computation of 3D spherical Fourier harmonic descriptorsA complete orthonormal basis for a rotational invariant representation of three-dimensional objects," in Proc. 12th IEEE Int. Conf. Comput. Vis. Workshops (ICCV). Kyoto, Japan, Sep./Oct. 2009, pp. 1863-1869.

[48] R. Marchant and P. Jackway, "Modelling line and edge features using higher-order Riesz transforms," in Advanced Concepts for Intelligent Vision Systems (Lecture Notes in Computer Science), vol. 8192, J. Blanc-Talon, A. Kasinski, W. Philips, D. Popescu, and P. Scheunders, Eds. Springer, 2013, pp. 438-449.

[49] D. Slepian, "Prolate spheroidal wave functions, Fourier analysis, and uncertainty-V: The discrete case," Bell Syst. Tech. J., vol. 57, no. 5, pp. 1371-1430, 1978.

[50] R. Bellman, "Dynamic programming and Lagrange multipliers," in The Bellman Continuum: A Collection of the Works of Richard E. Bellman. Singapore: World Scientific, 1986, p. 49.

[51] M. D. Abràmoff, P. Magalhães, and S. J. Ram, "Image processing with ImageJ," Biophoton. Int., vol. 11, no. 7, pp. 36-42, Jul. 2004.

[52] D. Sage, F. R. Neumann, F. Hediger, S. M. Gasser, and M. Unser, "Automatic tracking of individual fluorescence particles: Application to the study of chromosome dynamics," IEEE Trans. Image Process., vol. 14, no. 9, pp. 1372-1383, Sep. 2005.

[53] B. Pesquet-Popescu and J. L. Vehel, "Stochastic fractal models for image processing," IEEE Signal Process. Mag., vol. 19, no. 5, pp. 48-62, Sep. 2002.

[54] A. P. Pentland, "Fractal-based description of natural scenes," IEEE Trans. Pattern Anal. Mach. Intell., vol. PAMI-6, no. 6, pp. 661-674, Nov. 1984.

[55] J. Fischbarg, "Fluid transport across leaky epithelia: Central role of the tight junction and supporting role of aquaporins," Physiol. Rev., vol. 90, no. 4, pp. 1271-1290, 2010.

[56] J. Makhoul, "On the eigenvectors of symmetric Toeplitz matrices," IEEE Trans. Acoust., Speech Signal Process., vol. 29, no. 4, pp. 868-872, Aug. 1981.

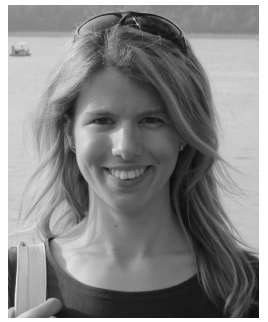

Zsuzsanna Püspöki was born in Budapest, Hungary, in 1986. She received the M.Sc. Diploma (Hons.) degree in electrical engineering from the Budapest University of Technology and Economics, in 2011. She is currently pursuing the Ph.D. degree with the Biomedical Imaging Group, École polytechnique fédérale de Lausanne, Switzerland, under the direction of M. Unser. She is currently working on problems related to image analysis, wavelet theory, and computer vision. Her research interests include the analysis of local geometric transformations and symmetries, and directional analysis in images.

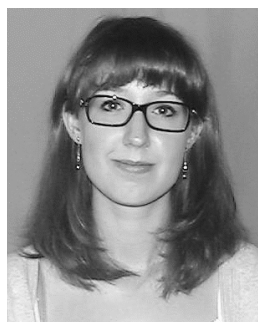

Virginie Uhlmann was born in Nyon, Switzerland, in 1989. She received the M.S. degree in bioengineering from the École polytechnique fédérale de Lausanne (EPFL), Switzerland, in 2012, where she is currently pursuing the Ph.D. degree with the Biomedical Imaging Group under the direction of M. Unser. She pursued her master's thesis as a Visiting Student in the Imaging Platform with the Broad Institute, Cambridge, MA, under the supervision of A. Carpenter. She is working on applied problem related to image segmentation and tracking, and on approximation and spline theory. Her research interests include computer vision, machine learning, and life sciences. She received the Competitive Excellence Fellowship at the Master's level from EPFL from 2011 to 2012. She also received four prizes, including third best grade point average among EPFL's M.S. graduates of 2012.

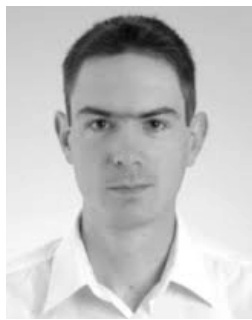

Cédric Vonesch was born in Obernai, France, in 1981. He received the M.S. degree from the École polytechnique fédérale de Lausanne (EPFL), Switzerland, in 2004, and the Ph.D. degree from the Biomedical Imaging Group, EPFL, under the direction of Prof. M. Unser, in 2009. He then joined the Program in Applied and Computational Mathematics, Princeton University, for a post-doctoral position. He joined the Biomedical Imaging Group, EPFL, in 2012. His primary research interests are inverse problems in the imaging sciences and multiresolution (wavelet-based) methods. He has worked on applications to fluorescence and electron microscopy, as well as seismic imaging.

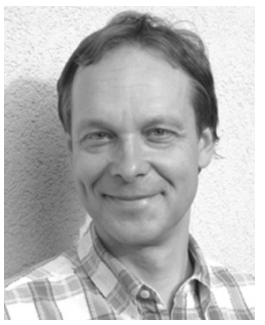

Michael Unser (M'89-SM'94-F'99) received the M.S. (summa cum laude) and Ph.D. degrees in electrical engineering from the École polytechnique fédérale de Lausanne (EPFL), Switzerland, in 1981 and 1984, respectively. From 1985 to 1997 , he was a Scientist with the National Institutes of Health, Bethesda, USA. He is currently a Full Professor and the Director of the Biomedical Imaging Group with EPFL. His main research area is biomedical image processing. He has a strong interest in sampling theories, multiresolution algorithms, wavelets, and the use of splines for image processing. He has published 200 journal papers in those topics and is one of ISI's highly cited authors in Engineering. He is a fellow of EURASIP and a member of the Swiss Academy of Engineering Sciences. He received the 1995 and 2003 Best Paper Awards, the 2000 Magazine Award, and two IEEE Technical Achievement Awards (2008 SPS and 2010 EMBS). He was the Associate Editor-in-Chief (2003-2005) of the IEEE TRANSACTIONS ON MEDICAL IMAGING, and an Associate Editor of the IEEE TRANSACTIONS ON MEDiCAL IMAGING (1999-2002; 2006-2007), the IEEE TRANSACTIONS ON IMAge Processing (1992-1995), and the IEEE Signal Processing LETTERS (1994-1998). He is currently a member of the Editorial Boards of Foundations and Trends in Signal Processing, and Sampling Theory in Signal and Image Processing. He co-organized the first IEEE International Symposium on Biomedical Imaging (2002) and was the Founding Chair of the Technical Committee of the IEEE-SP Society on Bio Imaging and Signal Processing. 